\title{
Detection of Natural Randomness by Chipless RFID Approach and its Application to Authentication
}

\author{
Zeshan Ali, Etienne Perret, Senior Member, IEEE, Nicolas Barbot, Romain Siragusa, David Hély, \\ Maxime Bernier, and Frédéric Garet
}

\begin{abstract}
In this paper, a chipless solution dedicated to the authentication of manufactured products is proposed. The proposed technique is an extension of chipless radio frequency identification (RFID) with a prevention of tags' cloning due to the use of natural randomness in the fabrication process. For the first time, we proposed a methodology to characterize the chipless RFID tags for the purpose of authentication. The proposed method has a high-level of security due to the employment of a database of authenticity. With a non-intrusive and non-destructive operation, the proposed method has promising possibilities for authentication applications, for example, the chipless tags can be inserted (or hidden) in the product packaging. The chipless tags are realized two times intermittently, where each realization is constituted of forty-five tags. These two different realizations share the same company, same technology, but different film mask to ensure the natural dimensional randomness. The similarity analysis is conducted inside each realization and also between two different realizations. With the help of this technique, it is possible to authenticate two chipless RFID tags exhibiting natural dimensional variations in their designs with a low probability of error.
\end{abstract}

Index Terms-Anti-counterfeiting, authentication, C-folded scatterer, chipless RFID, fabrication tolerance, randomness.

\section{INTRODUCTION}

$\mathrm{P}$ RODUCT authentication offers vast opportunities to combat fakes in the global supply chain. For this purpose, numerous anti-counterfeit technologies have been reported in the literature which can be generally classified into three categories [1], [2]: overt or visible features (security level 1), covert or hidden markers (security level 2), and forensic techniques (security level 3). For security levels 1 and 2, a

This work was supported in part by the University of Grenoble Alpes via the AGIR Program, in part by the Région Auvergne-Rhône-Alpes via the ARC6 Program, and in part by the Institut Universitaire de France.

Zeshan Ali, Nicolas Barbot, Romain Siragusa, and David Hély are with the University of Grenoble Alpes, Grenoble INP, LCIS, 26000 Valence, France (e-mail: zeshan.ali@ lcis.grenoble-inp.fr).

Etienne Perret is with the University of Grenoble Alpes, Grenoble INP, LCIS, 26000 Valence, France, and also with the Institut Universitaire de France, 75005 Paris, France.

Maxime Bernier and Frédéric Garet are with the University Savoie Mont Blanc, CNRS, Grenoble INP, IMEP-LAHC, 73376 Le Bourget-du-Lac, France. database of authenticity might not be needed and authentication can be performed by the observation of specific features of authentication elements. To elevate the security level of an authentication technique, a database of authenticity might be employed that necessitate a comparison between the measurements at the instant of authentication with the measurements stored in the database. For this reason, a database in security level 3 is very important for the highly secure application.

In the literature, conventional passive radio frequency (RF) identification (RFID) technology [3] is also utilized for authentication solutions. Strictly speaking, a conventional RFID tag cannot be directly employed for authentication because it is susceptible to be copied easily. Authentication differs from identification because authentication identifies as well as determines the genuineness of its subject. One way to employ conventional RFID for the authentication applications is to use the analog features (physical attributes) of backscattered signals from the RFID tags [4]-[12]. In the context of RF fingerprinting, several approaches to extract the fingerprints have been reported [4]-[7]. In these approaches the fingerprints are based on, for example, the minimum power responses of RFID tags [4], the wavelet fingerprinting [5], the nanostructured composite materials [6], and the phase information of back-scattered signals [7]. On the other hand, some studies on near-field physical-layer identification have also been reported in the literature [8]-[12]. Here, the fingerprints are based on, for example, the magnitude and the phase information [8], the modulation shape and the spectral features [9], the principal components analysis [10], the frequency of resonance and the quality factor [11], and the normalized power spectral density of envelop of backscattered signals [12].

Chipless RFID has also been used in authentication. Here, the authentication has been done through the medium of dipoles [13], a plurality of conductive wires [14] and a barcode that exhibits an electromagnetic (EM) signature [15]. On the other hand, near-field RFID systems [16], [17] have also been presented for secure proximate field data transfer (or authentication). Some other approaches such as RF absorbing markers [18] and tag-less RFID approach [19] have also been discussed.

Natural randomness during the fabrication process is 
inevitable up to a certain degree. Such randomness can be exploited to generate unique EM signature for each realization. These unique signatures can then be utilized for authentication applications. In this context, the simulation results of a chipless RFID system have been discussed in [20] to present an unclonable RFID tag. Other examples of the authentication systems based on the natural randomness are the RF certificates of authenticity (RF CoA) [21]-[23], and a plurality of randomly arranged resonators [24]. To exploit the natural texture of products' surface (e.g., the arrangement of paper fibers), an optical technique is presented in [25].

In previous recent works [26]-[28], we have also demonstrated that apart from the identification applications, the chipless RFID technology can be extended to the unitary product authentication applications. Our previous work is summarized as follows: first, a tiny population of the chipless tags (i.e., three tags) has been designed by purposely applying the dimensional variations to the less effective geometrical dimension parameters (the width of the arms and the spacing between two arms) of classical C-folded scatterers. It is important to note that the design of employed classical chipless tags is without any optimization on the shape for the purpose of authentication. Then, the idea of chipless tag discrimination has been presented using the cavity resonator measurements [26] and the free space measurements [27]. Subsequently, a moderate population of the chipless tags (i.e., five tags for three groups = fifteen tags) is designed, where the dimensional variations are purposely applied to the most effective geometrical dimension parameters (the length of arms) of optimized C-folded scatterer [28]. The prime focus in this work is the modeling and detection of such purposely applied variations by a second-order bandpass filter model.

The techniques of the security levels 1 and 2 (overt and covert features) might easily be mimicked. In the security level 3, the forensic techniques might be invasive or destructive in operation. Also, the necessity to use lab equipment is a drawback. These are very expensive solutions that might not be possible to implement to the low-cost items for the authentication application. The authentication techniques based on the chip-based RFID [4]-[12] might be bulky or costly due to the employment of chip. In the category of natural randomness based chipless approaches, the techniques based on RF CoA [21]-[23] might require 3D structures. The technique presented in [24] might require a particular printing procedure. The optical technique [25] might have a limitation of close imaging procedure, that can also be imitated.

In this paper, chipless authentication based on solely naturally occurring randomness (i.e., without any purposely applied variations) in the printed circuit board (PCB) is presented. The natural randomness along geometrical dimensions of a microstrip circuit vitally depends on the technology selected for circuit realization. For the chemical etching based PCB realization technology, non-systematic under- and over-etching are among the fabrication process anomalies [29], [30] which give rise to the natural process variations up to a slight extent (which is in the order of the metal thickness). For the first time in comparison with state of the art, we proposed that the PCB realizations of simple scatterers can be used as unique footprints. The naturally occurring randomness can be explained by the arrangement of paper fibers. Fig. 1(a) shows the scanning electron microscope (SEM) photograph of ordinary paper. The unique arrangement of paper fibers happens during the realization process naturally. To copy such naturally occurring pattern in an exact manner is virtually impossible. Similar kind of natural randomness exists in the realization process of PCB chipless tags as shown in Fig. 1(b). Therefore, the cloning of chipless tags in the proposed method is very difficult, as the natural dimensional variations in the chipless tags' realizations give rise to the unique variations in RF field that is compatible with the high-level security.

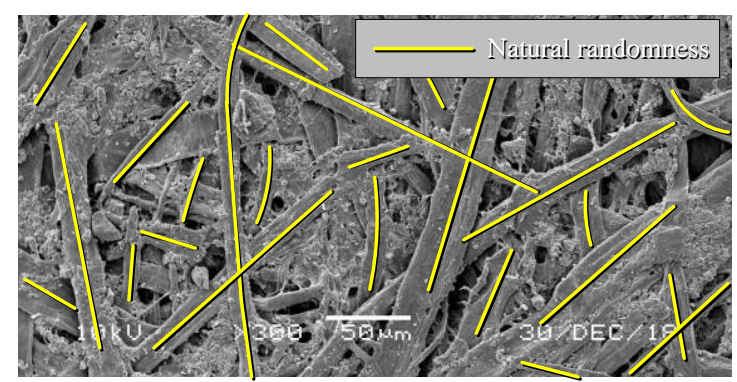

(a)

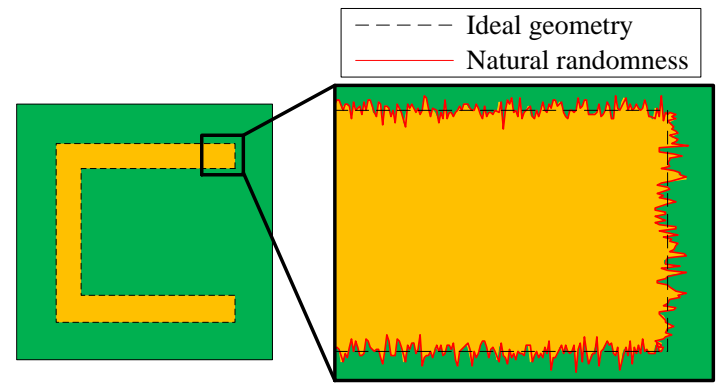

(b)

Fig. 1. Natural randomness in the realization process. (a) The unique random arrangement of paper fibers. (b) Illustration of natural process variations of geometrical dimensional of chipless RFID tags during the PCB realization.

However, the proposed concept of authentication using the natural randomness within PCB realizations is much broader than the related example of paper fibers, because numerous factors can play their role for the natural variations of $\mathrm{RF}$ signatures back-scattered by PCB realizations: the film mask, the chemical etching process, and the substrate. To implement the proposed concept, a population of chipless tags can be realized on one sample of substrate using one film mask or on different samples of substrates using different film masks. The comparison among the measurements of two chipless tags from a population of chipless tags which are realized on one sample of substrate using one film mask is referred to as the inter-tag. The comparison among the measurements of two chipless tags from a population of chipless tags which are realized on different samples of substrates using different film masks is referred to as the inter-realization. The inter- 
realization comparison tends to produce a larger magnitude of discrimination than the inter-tag comparison because of the contribution of randomness from the film mask as well as the substrate samples. The comparison among the repetitive measurements of a single chipless tag is referred to as the intra-tag. This intra-tag comparison for each tag should reflect a high magnitude of similarity which shows the insignificance of measurement error. These conventions of signals' comparisons (i.e., the intra-tag, the inter-tag, and the interrealization) are explained graphically later in this paper (see Section II-B).

The proposed approach is very simple because we do not add any additional particle into the labels as done in [15]. It is important to note that our previous works [26]-[28] are based on the purposely applied variations but not the natural dimensional variations. For these reasons, our work can be differentiated from the existing works in the literature [15], [26]-[28].

First, we have proposed a methodology to characterize the chipless RFID tags for the purpose of authentication. Then, we have proved the concept with sufficient large population of chipless tags (i.e., forty-five tags). It has been discussed in [31] that the early part of time domain (TD) back-scattered response from a chipless RFID tag is a function of the polarization, the direction, and the distance between the tag and antenna. For this reason, this early part of TD backscattered response is referred to as the aspect-dependent information. On the other hand, the complex natural resonance (which analogously represents the frequency of resonance $f_{r}$ and the quality factor $Q$ ) is aspect-independent that is dependent only on the geometrical dimensions of scatterer. In this work, for similarity comparison between PCB realizations, the aspect-dependent part of measurements has been discarded and merely the signal part containing the aspect independent parameters (i.e., the frequency of resonance $f_{r}$ and the quality factor $Q$ ) is used. An in-depth statistical study has been presented to provide an accurate idea of the performance that can be reached. The natural dimensional variations in the realizations of chipless tags are characterized using microscopic analysis. Then, a relationship between the natural dimensional variations and the similarity variations (that are due to the aspect independent parameters) is established. Finally, the generalization of the proposed approach is presented. This paper is organized as follows. Section II describes the methodology of the proposed approach. Section III presents the design and the optimization of the chipless RFID tags to be employed for authentication. Section IV describes the detection of natural randomness, the authentication results and the generalization of the proposed method. Finally, Section VI draws the conclusions.

\section{Methodology}

The proposed chipless authentication is a highly secure application, due to the comparison of a given measurement with the database of pre-stored measurements at the time of authentication. This Section discusses the procedure for the authentication and the statistical analysis.

\section{A. Authentication Procedure}

The principle of authentication procedure is explained in Fig. 2. The employment of chipless tags for authentication can be divided into two stages: the formation of database (prestage) and the comparison of a given unknown tag with the database (post-stage). Fig. 2(a) shows the method of formation of database, where each chipless tag is measured individually in a controlled anechoic environment (e.g., box-like geometry) using a chipless RFID reader. Then, the measurements are saved in a database.

Next, the comparison procedure to check a unique match of a given unknown chipless RFID tag from the database is depicted in Fig. 2(b). Prior to the comparison, the signals are post-processed (i.e., background normalization and time windowing). The details of post-processing can be seen in [27], [28]. Then, the post-processed signal from an unknown test tag is compared with all post-processed signals from the database to find a possible unique match. The proposed comparison can be performed in the frequency domain (FD) using cosine similarity (CS) or in the TD using maximum value of correlation coefficient $\left(\mathrm{CC}_{\max }\right)$ as presented in [27]. The mathematical expression of CS is:

$$
\mathrm{CS}=\left|\frac{\sum\left(\mathrm{u}_{i} \cdot \mathrm{v}_{i}^{*}\right)}{\sqrt{\sum\left(\mathrm{u}_{i}\right)^{2}} \sqrt{\sum\left(\mathrm{v}_{i}\right)^{2}}}\right|
$$

where $\mathrm{u}=\left[u_{1}, u_{2}, \ldots, u_{\mathrm{n}}\right]$ and $\mathrm{v}=\left[v_{1}, v_{2}, \ldots, v_{\mathrm{n}}\right]$, are two EM signals to be compared, exhibiting complex and discrete spectrums. The operator * represents the complex conjugate.

The mathematical expression of $\mathrm{CC}_{\max }$ is:

$$
\mathrm{CC}_{\max }=\max \left[\frac{\sum_{i}\left(\mathrm{n}_{i}-\mu_{\mathrm{n}}\right)\left(\mathrm{m}_{i}-\mu_{\mathrm{m}}\right)}{\sqrt{\sum_{i}\left(\mathrm{n}_{i}-\mu_{\mathrm{n}}\right)^{2}} \sqrt{\sum_{i}\left(\mathrm{~m}_{i}-\mu_{\mathrm{m}}\right)^{2}}}\right]
$$

where $\mathrm{n}=\left[n_{1}, n_{2}, \ldots, n_{\mathrm{n}}\right]$ and $\mathrm{m}=\left[m_{1}, m_{2}, \ldots, m_{\mathrm{n}}\right]$ are two time-series EM signals to be compared. The $\mu_{\mathrm{n}}$ and $\mu_{\mathrm{m}}$ denote the arithmetic means of two time-series EM signals, respectively.

After the comparison of the signal from an unknown tag with the database, the maximum value of resultant vector can be compared with an optimum similarity threshold value $x_{\text {opt }}$ to check the authenticity of tag. This optimum similarity threshold value $x_{\text {opt }}$ should be selected at the minimum probability of error (PE) of the system, as it is a critical parameter that defines the performance of our approach. The calculation of this parameter $x_{\text {opt }}$ is discussed later in this Section.

To deploy the proposed method, a controlled anechoic environment is required for the measurements. This controlled environment can be realized within a small box with free volume of $30 \times 30 \times 30 \mathrm{~cm}^{3}$ after placing the absorbents. This free volume of $30 \times 30 \times 30 \mathrm{~cm}^{3}$ is suggested because the typical read range of chipless RFID tags is around $15 \mathrm{~cm}$. To avoid direct reflections from the walls, the most significant aspect is to select the absorbents compatible with the frequency range of impulse signal. In this way, the height of absorbents is dependent on the frequency range of impulse 
signal. As a consequence, the overall size of box is dependent on the height of employed absorbents. With such a fixed measurement environment, the measurement parameters (e.g., the distance between antennas if the setup is in bistatic configuration, and the distance between the tag and the antenna) always remain consistent. This implies that the time windowing parameters (i.e., the part of post-processing) will also be consistent.

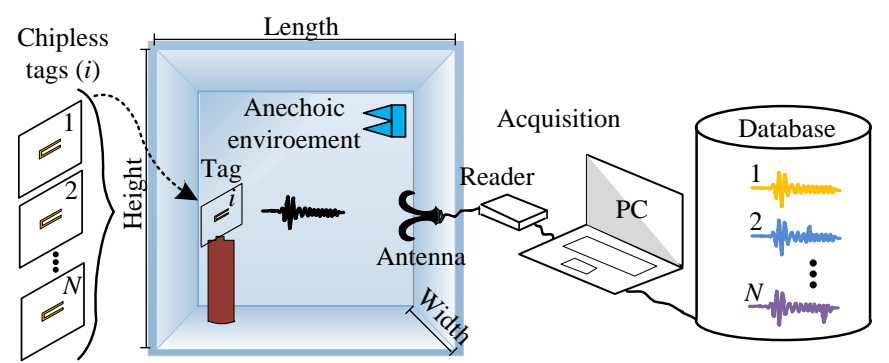

(a)



(b)

Fig. 2. Principle of the authentication procedure. (a) Pre-stage: the formation of database. (b) Post-stage: the comparison of a given unknown tag with the database.

From the literature, numerous biological and digital authentication techniques might be considered at security level 3 since these are machine readable techniques exhibiting specific features and/or equipment for authentication. From our viewpoint, a database of authenticity is a primary requirement for an authentication technique to be a highly secure application. Therefore, an authentication approach without such a database based comparison might not be considered as an application of level 3 of security. The application of the proposed approached can be considered as a highly secure security seal (but not only limited to this), where the tag can be inserted (or hidden) in the corrugated layers or the pulp of product packing. At the time of authentication, the tag can be taken out by the authorized person to check its authenticity with the procedure as explained in Fig. 2. This authorized extraction of the tag does not make the proposed authentication a destructive method, as the tag is not destroyed and still reusable.

\section{B. Statistical Analysis}

In-depth statistical analysis is used to analyze the performance of the proposed method. Fig. 3 presents the concept of statistical analysis of the proposed approach. The intra-tag, the inter-tag, and the inter-realization comparisons are graphically explained in Fig. 3(a). For all comparisons with all possible combinations among the signals of chipless tags, the histogram distributions are constructed. These histogram distributions can be of any form or shape, as the comparison data is based on a random source. We estimated the probability density function (PDF) from the histogram distribution by using a normalization, where the frequency counts are divided by the area under the histogram. Ideally, the central tendency (e.g., the mean value) of the intra-tag PDF $f_{\text {intra-tag }}$ should be towards a value of 1 , as this PDF is based on the comparison among repetitive signals of each individual tag from the population. Therefore, an intra-tag PDF centered around a value 1 shows the repeatability of the system (i.e., measurement error is less than natural random variations). On the other hand, the ideal central tendency (e.g., the mean value) of the inter-tag PDF $f_{\text {inter-tag }}$ or the interrealization PDF $f_{\text {inter-realization }}$ should be towards a value of 0 . However, the individual tags realized on a single PCB share some properties (e.g., the substrate). Hence the back-scattered signals from them might not be totally independent. For this reason, $f_{\text {inter-tag }}$ is placed far from zero in Fig. 3(a). While $f_{\text {inter- }}$ realization is placed close to zero as compared to $f_{\text {inter-tag }}$ in Fig. 3(a), as the tags realized on different PCBs are less correlated. For the sake of simplicity, the intra-tag and the inter-tag PDFs are depicted only for realization 1 in Fig. 3(a). However, of course, these PDFs can also be constructed for realization 2 .

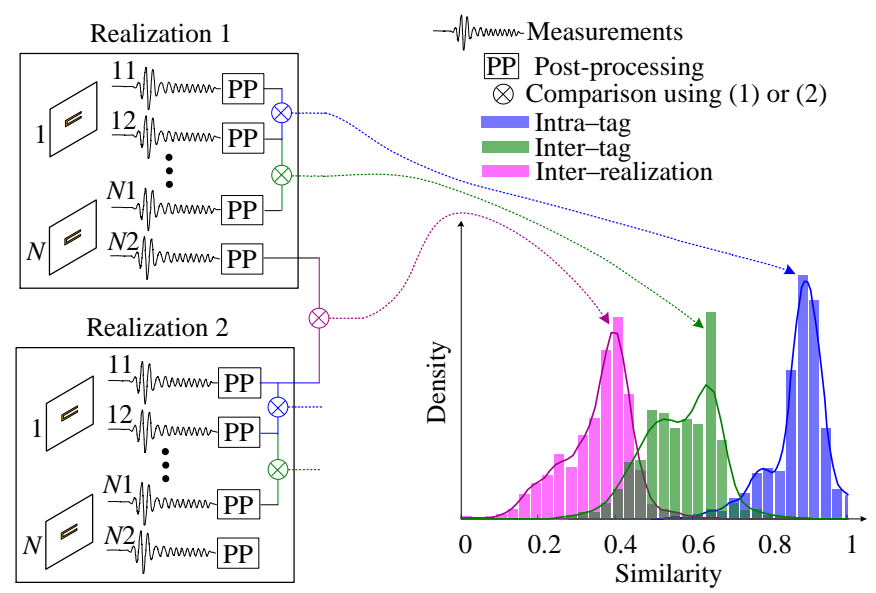

(a)

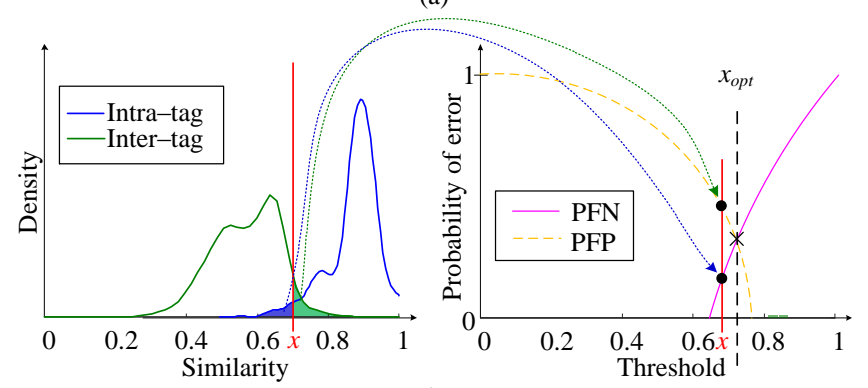

(b)

Fig. 3. Concept of statistical analysis of the proposed approach. (a) Procedure for the construction of similarity PDFs. Also, the description of signals' comparison conventions. (b) Procedure for the calculation of the error rates and the optimum similarity threshold value $x_{\text {opt }}$.

Fig. 3(b) presents the procedure for the calculation of the error rates and the optimum similarity threshold value $x_{\text {opt }}$ 
(used in the authentication procedure above). For the sake of simplicity, an example for one chosen realization is demonstrated, where we have chosen the intra- and inter-tag PDFs from Fig. 3(a) to Fig. 3(b).

The probability of false negative (PFN) is calculated from the intra-tag PDF $f_{\text {intra-tag }}$ using the following expression:

$$
\mathrm{PFN}=\int_{-\infty}^{x} f_{\text {intra-tag }}(x) d x
$$

where $x$ corresponds to the threshold.

The probability of false positive (PFP) is calculated from the inter-tag $\mathrm{PDF} f_{\text {inter-tag }}$ using the following expression:

$$
\mathrm{PFP}=\int_{x}^{+\infty} f_{\text {inter-tag }}(x) d x
$$

where $x$ corresponds to the threshold. We have presented examples of the calculation of PFN and PFP from the intra-tag and the inter-tag PDFs with threshold value $x$ at an assumed position in Fig. 3(b) [see the blue dotted line from the intra-tag PDF $f_{\text {intra-tag }}$ to PFN curve (solid magenta line) and see the green dotted line from the inter-tag PDF $f_{\text {inter-tag }}$ to PFP curve (dashed orange line)]. In practice, the threshold value $x$ is taken from 0 to 1 with 10000 points to calculate whole PFN and PFP curves.

The comparison between two PCB realizations is not shown in Fig. 3(b). In such inter-realization case, the intra-tag PDFs from both realizations are merged into a single PDF $f_{\text {inter-tag }}$ and then PFN is calculated using (3). On the other hand, PFP is calculated by using $f_{\text {inter-realization }}$ instead of $f_{\text {inter-tag }}$ in (4).

Finally, the PE and the optimum similarity threshold value $x_{\text {opt }}$ are computed from the intersection point of PFN and PFP [see cross symbol and black dashed line in Fig. 3(b)].

\section{DESIGN AND OPTIMIZATION OF CHIPLESS RFID TAGS TO BE EMPLOYED FOR AUTHENTICATION}

In this section, we have explained that a classical chipless RFID tag is not sensitive enough to be employed for the authentication. Therefore, an optimization to enhance the sensitivity of chipless tag should be done. This fact is explained by the simulations and the measurements of chipless tags exhibiting the purposely applied dimensional variations. Finally, a second-order bandpass filter model is utilized to create 2D similarity maps. With the help of 2D similarity maps, the performance of classical chipless RFID tags is compared with the performance of optimized chipless RFID tags.

In this work, we have chosen a widespread conventional chemical etching based PCB realization technique (that is known and proven) to give an accurate reference of what can be achieved by employing a chipless approach in authentication. Other realization technology such as inkjet printing might exhibit higher randomness due to an error in the placement of ink droplets. The sensitivity towards the naturally occurring randomness during the fabrication process and the simplicity in the design of a chipless tag to be employed for authentication application are the key and necessary characteristics. The C-folded dipole is simple in design that can be realized with a lot of different techniques (some of them can be low cost). This planar scatterer has been used extensively in the field of chipless RFID technology [32]. The frequency of resonance $f_{r}$ of such a resonator can be calculated using the following expression:

$$
f_{r}=\frac{c}{4 L^{\prime} \sqrt{\varepsilon_{e f f}}}
$$

where $L^{\prime}$ is the total length which is the sum of $L$ (physical length of each arm) and $\Delta L$ (complementary length added due to the fringing fields), and $\varepsilon_{\text {eff }}$ is the effective permittivity of the substrate. For a coplanar stripline [33], $\varepsilon_{\text {eff }}$ depends on various parameters including the spacing between two arms $g$, the trace width $w$, the metal thickness $t$, the substrate thickness $h$, and the permittivity of substrate $\varepsilon_{r}$. However, if the substrate parameters such as $t, h$, and $\varepsilon_{r}$ are fixed, the response of a $\mathrm{C}$-folded dipole is determined by two key dimensional parameters [see Fig. 4(a)]: the length $L^{\prime}$ and the spacing between two arms $g$. For the rest of this paper, we used $L^{\prime}$ and $L$ interchangeably for the ease in discussion.

\section{A. C-Folded Uni-Scatterer Tags (Classical Design)}

Fig. 4(a) shows the layout of a simulated chipless tag based on C-folded uni-scatterer. For simulations, a commercial fullwave simulator (CST Microwave Studio) is used, where the chipless tags are illuminated by a plane wave. The nominal geometrical dimensions of this simulated scatterer are $L=3.16 \mathrm{~mm}, g=2.5 \mathrm{~mm}$, and $w=0.22 \mathrm{~mm}$, while the overall size of tag is $8 \times 6 \mathrm{~mm}^{2}$. Concerning the value of $g$, it has been discussed in [34] that the radar cross section (RCS) level is increasing almost linearly with $g$. Therefore, to increase the RCS level, a higher value of $g=2.5 \mathrm{~mm}$ is taken. For nominal dimensional parameters, simulated CST backscattered TD and FD responses are shown in Fig. 4(b) and (c), respectively. It is known that a back-scattered field from a scatterer constitutes two modes: the early part of the signal called the structural mode and the late part of the signal called the antenna mode. Between these two modes, the structural mode is unessential in our work due to its association with the direct optical reflections from the tag and tag's support, while the antenna mode is essential as it is related to the reflections due to the resonance of scatterer. For this reason, the time windowing [27], [28] is applied to extract the late part of the signal corresponding to the antenna mode [see Fig. 4(b) and (c)].

It has been discussed in [28] that if the back-scattered TD response is comprising merely the antenna mode extracted by applying the time windowing to TD response, a second-order bandpass filter analytical model can also be employed to model such a kind of $\mathrm{C}$-folded scatterer:

$$
T(\omega)=G\left[\frac{\frac{2 m j \omega}{\omega_{0}}}{1+\frac{2 m j \omega}{\omega_{0}}+\left(\frac{j \omega}{\omega_{0}}\right)^{2}}\right]
$$


where $m$ and $\omega_{0}$ correspond to the damping ratio and the angular frequency of resonance, respectively, of a pole of second order. $G$ is the gain determining the amplitude level of back-scattered signal from the scatterer. To validate this analytical model (6), a time window of $3 \mathrm{~ns}$ is applied to TD signal in time windowing process to extract the antenna mode, where $2 \mathrm{~ns}$ of early part of signal is neglected to discard the structural mode [Fig. 4(b)]. The angular frequency of resonance $\omega_{0}$ and the damping ratio $m$ are extracted from the peak apex and the quality factor of FD windowed signal, respectively. The damping ratio $m$ is related to the quality factor $Q: m=1 /(2 Q)$. The quality factor $Q$ is computed by using $Q=f_{0} / \Delta f_{3 \mathrm{~dB}}$, where the frequency of resonance $f_{0}$ is extracted from the peak apex of FD windowed signal and the half power bandwidth $\Delta f_{3 \mathrm{~dB}}$ is extracted from $-3 \mathrm{~dB}$ level of FD windowed signal. Then, a model signal is generated using (6) with extracted $m$ and $\omega_{0}$ parameters. Subsequently, a fitting comparison among the non-windowed FD signal, the windowed FD signal and the model (6) generated signal is shown in Fig. 4(c). The response of the analytical model (6) is in perfect agreement with the simulated windowed FD signal. For the sake of simplicity, the signals are presented in normalized amplitude level (i.e., $G=1$ ). It is important to note that the extracted $m$ and $\omega_{0}$ parameters are aspect-independent parameters. For nominal geometrical dimensions of $\mathrm{C}$-folded uni-scatterer tag, the estimated values (extracted from CST simulation) of $f_{r}, Q$, and RCS level are $f_{r}^{\mathrm{CST}} \approx 12.22 \mathrm{GHz}$, $Q^{\mathrm{CST}} \approx 22.5$ and $\mathrm{RCS}^{\mathrm{CST}} \approx-35.7 \mathrm{dBsm}$, respectively.

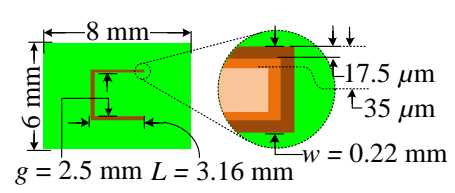

(a)

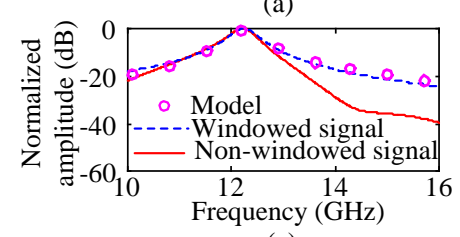

(c)

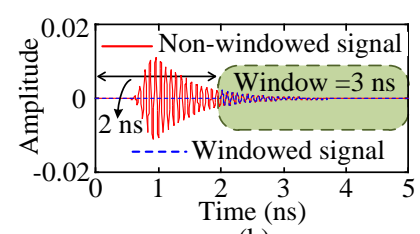

(b)

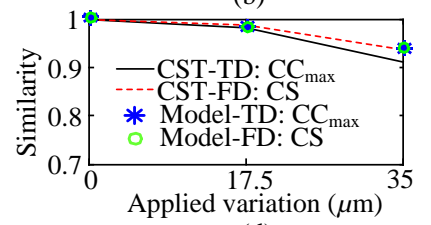

(d)
Fig. 4. (a) Layout of the simulated C-folded uni-scatterer tag. Also, the purposely applied variations along the trace are shown in different shades of copper color. (b) Simulated CST back-scattered TD responses. (c) Simulated CST back-scattered FD responses. Also, a comparison of the windowed FD signal with the signal generated by the analytical model (6). (d) Similarity level calculated in both FD (1) and TD (2) using the simulated CST backscattered responses as well as the model generated signals.

In this work, a high-performance substrate (Rogers RO4003) is used with $\varepsilon_{r}=3.38$, and $h=0.81 \mathrm{~mm}$. This substrate is in contrary to FR4 where $\varepsilon_{r}$ can change from one sample to another. For this reason, the natural changes to be used for authentication are not only linked to the substrate. Initially, to show the sensitivity of a $\mathrm{C}$-folded uni-scatterer chipless tag towards the naturally occurring randomness with the help of simulations, we have purposely applied variations along all sides of the metal trace as shown in different shades of copper color [see Fig. 4(a)]. These purposely applied variations are in the order of maximum probable fabrication tolerance, that is $t=35 \mu \mathrm{m}$, in the form a decrease of a step of $17 \mu \mathrm{m}$. It is important to note that the application of these purposely applied variations along all sides of the metal trace would affect $w$ as well as $g$ and $L$. The sensitivity of this Cfolded uni-scatterer tag is presented in Fig. 4(d). This sensitivity is calculated by comparing the signals as the result of applied dimensional variations with a reference signal. The reference signal is the one that is obtained at nominal dimensions. Prior to the signals' comparison, the time windowing is applied to all signals. Next, $m$ and $\omega_{0}$ parameters are extracted from all simulated windowed FD signals. Then, we generated the model signals by using (6) with the extracted $m$ and $\omega_{0}$ parameters. Finally, the model generated signals are also compared among them to calculate the sensitivity of analytical model (6). The signals' similarities ( $\mathrm{CS}$ and $\mathrm{CC}_{\max }$ ) are calculated by using (1) for FD signals and using (2) for TD signals as discussed in Section II. As expected, the decrease in similarity is happened because of applied dimensional variations [see Fig. 4(d)]. At the applied variation of $35 \mu \mathrm{m}$, the changes in similarity metrics (1) and (2) for both the simulated CST back-scattered and the model signals are $\Delta \mathrm{CS}_{1}{ }^{\mathrm{CST}}=0.06, \Delta \mathrm{CC}_{\max 1}{ }^{\mathrm{CST}}=0.09, \Delta \mathrm{CS}_{1}{ }^{\text {Model }}=0.06$, and $\Delta \mathrm{CC}_{\max 1}{ }^{\text {Model }}=0.06$, respectively. It can be observed that the similarity change calculated using all data points of the simulated CST back-scattered signals $\left(\Delta \mathrm{CS}_{1}{ }^{\mathrm{CST}}\right.$ and $\left.\Delta \mathrm{CC}_{\max 1}{ }^{\mathrm{CST}}\right)$ and the similarity change calculated using the model (6) signals $\left(\Delta \mathrm{CS}_{1}{ }^{\text {Model }}\right.$ and $\Delta \mathrm{CC}_{\max 1}{ }^{\text {Model }}$ that are generated by using merely two aspect-independent $m$ and $\omega_{0}$ parameters) are almost equal. It is clear that the significant information in the back-scattered signals is related to $m$ and $\omega_{0}$ parameters due to their aspect-independent nature.

As discussed above, the quality factor $Q$ is related to the damping ratio $m: Q=1 /(2 m)$. The quality factor $Q$ of such a C-folded theoretical scatterer, with a very small value of $g$, can also be calculated using the following expression [35]:

$$
\frac{1}{Q} \simeq\left(\frac{g}{\lambda}\right)^{2}
$$

To practically validate the sensitivity of such C-folded uniscatterer tags by experimental results, we have realized two groups of prototypes, where each group is constituted of five tags. For the PCB realization of group $\mathrm{N}$, the nominal dimensional parameters of simulated tag [see Fig. 4(a)] are used for the formation of its film mask. To apply a variation on the subsequent group, we shortened $L$ by $100 \mu \mathrm{m}$ in the design of film mask for group $M$. The purpose behind this large applied variation (as compared to $35 \mu \mathrm{m}$ ) is to clearly discriminate the chipless tags without any ambiguity (i.e., observational noise or the systematic noise). Here, it is important to note that the natural process variations during the PCB realization are considered as a part of purposely applied variations. A photograph of first tag from each group along with their dimensions is shown in Fig. 5. The overall size of these fabricated tags is also same as the simulated ones': 
$8 \times 6 \mathrm{~mm}^{2}$.

The measurement setup for C-folded uni-scatterer tags is shown in Fig. 6, where the measurements are done in a bistatic co-polarization configuration using a vector network analyzer (VNA; Agilent 5222A) with a source power of $-5 \mathrm{dBm}$ in an anechoic environment. The distance between the antennas and the tag is $r=16.5 \mathrm{~cm}$, while the distance between both antennas is $e=2.7 \mathrm{~cm}$.



Fig. 5. Top view of the fabricated C-folded uni-scatterer tags along with their dimensions.

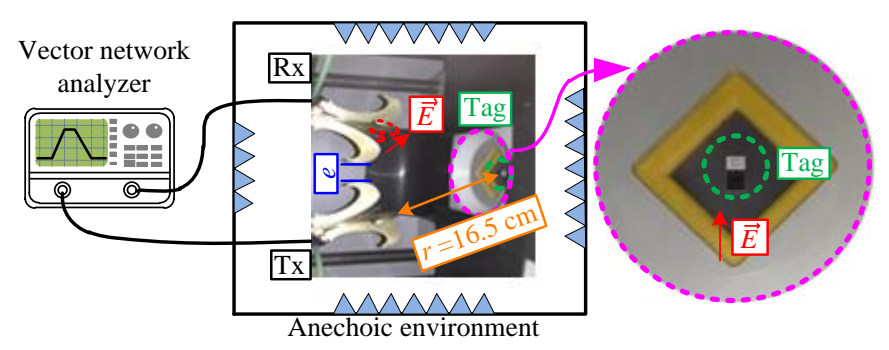

Fig. 6. Measurement setup for C-folded uni-scatterer tags in an anechoic environment with a bistatic co-polarization configuration.

The measured quantity is the transmission coefficient $S_{21}$ as shown in Fig. 7. For each tag, five repetitive measurements are taken. For each measurement trial, the tag is removed and repositioned at its position to ensure the validity of repeatability in the presence of an observational error. Furthermore, background normalization is applied (i.e., subtraction of an instantaneous measurement taken in the absence of tag from the measurement taken in the presence of tag). It can be observed that the measured non-windowed signal [see Fig. 7(b)] does not exhibit structural mode as compared to the simulated CST back-scattered signal [see Fig. 4(b)]. The reason behind this is measurements are done using bistatic co-polarization configuration, while in simulations an E-field probe is used (that is similar to monostatic co-polarization configuration). In such a case (bistatic measurement configuration) a significant part of structural mode is already removed due to the nature of configuration. So, the time windowing procedure (explained in [27]) is not needed to discard the structural mode. To explain this fact, a time window of $10 \mathrm{~ns}$ is employed to extract the antenna mode, where $1.6 \mathrm{~ns}$ of early part of signal is discarded to neglect the minor structural mode [Fig. 7(b)]. In Fig. 7(a), it can be observed that both FD non-windowed and FD windowed signals do not differ a lot from each other, in contrary to simulated CST back-scattered FD signals in Fig. 4(c).

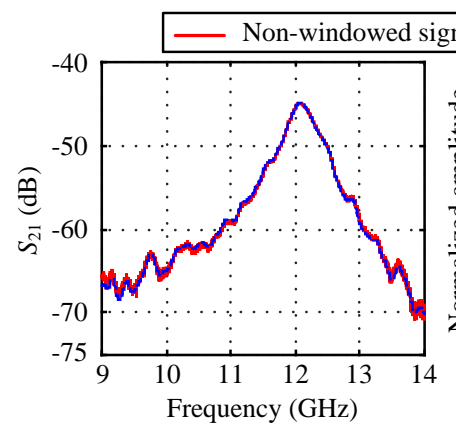

(a)



(b)
Fig. 7. Time windowing of the transmission coefficient $S_{21}$ of C-folded uniscatterer tag N1. (a) Frequency domain responses. (b) Time domain responses.

A CS map for uni-scatterer tags is presented in Fig. 8. To construct this map, we extracted $f_{r}$ and $Q$ parameters of all twenty-five measurements related to each group. The mean values of the extracted $f_{r}$ and $Q$ parameters of group $\mathrm{N}$, $f_{r}^{m}=12.05 \mathrm{GHz}$ and $Q^{m}=27$ are taken as a reference. First, a reference signal $x^{r}$ is generated by using the analytical model (6) with the reference $f_{r}^{m}$ and $Q^{m}$ parameters. Then a varying signal $x^{v}$ is generated by using the analytical model (6) by varying $f_{r}^{m}$ and $Q^{m}$ parameters such that $f_{r}^{v}=f_{r}^{m}-0.2 \mathrm{GHz}$ to $f_{r}^{v}=f_{r}^{m}+1 \mathrm{GHz}$ and $Q^{v}=Q^{m}-10$ to $Q^{v}=Q^{m}+100$. Subsequently, the reference signal $x^{r}$ and the varying signal $x^{v}$ are compared in the form of complex FD signals using CS (1). Finally, CS score is plotted versus $\Delta f_{r}$ and $\Delta Q$ such that $\Delta f_{r}=f_{r}^{v}-f_{r}^{m}$ and $\Delta Q=Q^{v}-Q^{m}$. Furthermore, the extracted $f_{r}$ and $Q$ parameters related to all twenty-five measurements of each group are superimposed on the similarity map for an intuitive comparison. The centroid to centroid difference of CS between groups $\mathrm{N}$ and $\mathrm{M}$ is $\Delta \mathrm{CS}_{1}=0.13$. This $\Delta \mathrm{CS}_{1}=0.13$ is large as compared to the simulated $\Delta \mathrm{CS}_{1}{ }^{\mathrm{CST}}=0.06$ [see Fig. 4(d)]. The reason of this difference between $\Delta \mathrm{CS}_{1}$ and $\Delta \mathrm{CS}_{1}{ }^{\mathrm{CST}}$ is that in simulations a uniform dimensional variation of $35 \mu \mathrm{m}$ is applied along all sides of metal trace, while in measurements a dimensional variation of $100 \mu \mathrm{m}$ is applied merely along $L$. It is important to mention that the natural dimensional variations happened during the realization of uni-scatterer tags are considered as a part of applied dimensional variations. Fig. 8 is a useful representation that can provide direct information of the potential performance (e.g., the slope of CS) of chipless RFID technology for authentication.

\section{B. C-Folded Quad-Scatterers Tags (Optimized Design)}

From a practical viewpoint, the C-folded uni-scatterer with a low $Q$ factor and a low RCS level of metallic resonator requires a bistatic measurement apparatus with two antennas. Therefore, we have coupled four scatterers to realize one tag with a higher $Q$ to make it appropriate in practical application. The influence of a high $Q$ chipless tag in authentication is discussed next. Fig. 9(a) shows the layout of a simulated chipless tag based on C-folded quad-scatterers. In this quadscatterers design, the nominal geometrical dimensions of each C-folded scatterer is exactly same as the geometrical dimensions of $\mathrm{C}$-folded scatterer of uni-scatterer tag (see 
Fig. 5), while the spacing among scatterers is $s=0.15 \mathrm{~mm}$ and the overall size of tag is $8 \times 15 \mathrm{~mm}^{2}$. For the nominal dimensional parameters, the simulated CST back-scattered TD and FD responses are shown in Fig. 9(b) and (c), respectively. Time windowing is employed by using a time window of $10 \mathrm{~ns}$ to extract the antenna mode while 2 ns of early part of signal is neglected to discard the structural mode. From the windowed signals [Fig. 9(b) and (c)], $\omega_{0}$ and $m$ are extracted to generate a model signal from the analytical model (6). Subsequently, a fitting comparison between the windowed FD signal and the model (6) generated signal is shown in Fig. 9(c). It can be observed that the response of analytical model is in perfect agreement with the simulated windowed FD signal.

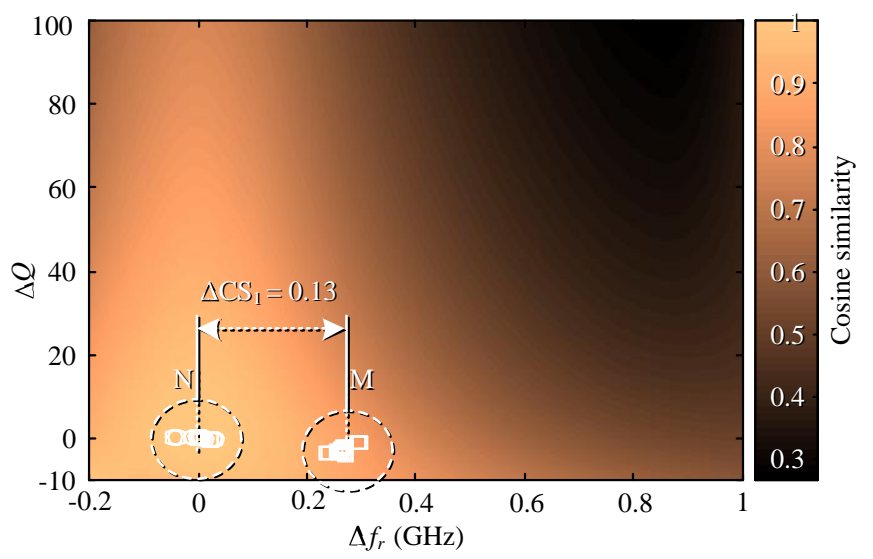

Fig. 8. Cosine similarity map obtained by comparing a reference complex FD signal $x^{r}$ with a varying complex FD signal $x^{v}$ using CS (1) for uni-scatterer tags. The superimposed markers $\bigcirc$ and $\square$ correspond to extracted parameters of twenty-five measurements of five tags of $\mathrm{N}$, and $\mathrm{M}$ groups, respectively.

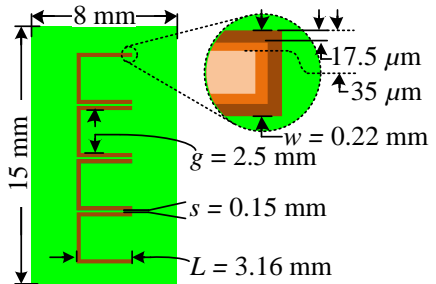

(a)

(c)



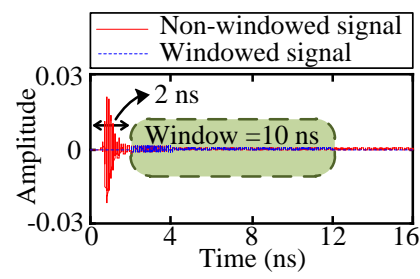

(b)

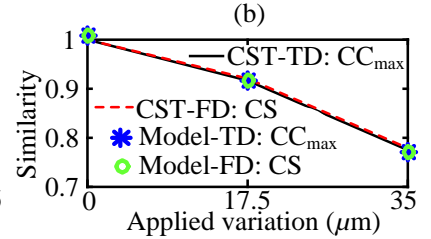

(d)
Fig. 9. (a) Layout of simulated C-folded quad-scatterers tags. Also, the purposely applied variations on the trace are shown in different shades of copper color. (b) Simulated CST back-scattered TD responses. (c) Simulated CST back-scattered FD responses. Also, a comparison of the windowed FD signal with a signal generated by the analytical model (6). (d) Similarity level calculated in both FD (1) and TD (2) using the simulated CST back-scattered responses as well as the model generated signals.

Like the uni-scatterer tag as discussed above [see Fig. 4(a)], we have also applied variations purposely along all sides of metal trace shown in different shades of copper color [see
Fig. 9(a)] to check the sensitivity of such C-folded quadscatterers based tag towards the applied geometrical variations (i.e., in the order of fabrication tolerance) in simulations. The simulated CST back-scattered signals and the model generated signals are compared [as discussed above for Fig. 4(d)] in FD (1) and TD (2) to calculate the sensitivity of quad-scatterers tags as shown in Fig. 9(d). At the applied variation of $35 \mu \mathrm{m}$, the changes in similarity metrics (1) and (2) for both the simulated CST back-scattered and the model signals are $\Delta \mathrm{CS}_{4}{ }^{\mathrm{CST}}=0.22, \Delta \mathrm{CC}_{\max 4}{ }^{\mathrm{CST}}=0.23, \Delta \mathrm{CS}_{4}{ }^{\text {Model }}=0.23$, and $\Delta \mathrm{CC}_{\max }{ }^{\text {Model }}=0.23$, respectively. It can be observed that the similarity change calculated using all data points of CST backscattered signals $\left(\Delta \mathrm{CS}_{4}{ }^{\mathrm{CST}}\right.$ and $\left.\Delta \mathrm{CC}_{\max 4}{ }^{\mathrm{CST}}\right)$ and the similarity change calculated using the model (6) signals $\left(\Delta \mathrm{CS}_{4}{ }^{\text {Model }}\right.$ and $\Delta \mathrm{CC}_{\max 4}{ }^{\text {Model }}$ that are generated by using merely two aspectindependent $m$ and $\omega_{0}$ parameters) are almost equal. Again, this behavior highlights the significance of aspect-independent $m$ and $\omega_{0}$ parameters.

Similar to the C-folded uni-scatterer tags, the sensitivity of such $\mathrm{C}$-folded quad-scatterers tags is demonstrated by experimental results. For this purpose, we fabricated two groups of prototypes for quad-scatterers tags, where each group is constituted of five tags. For the PCB realization of group N, its film mask is formed using the nominal dimensional parameters of simulated tag as depicted in Fig. 9(a). Like the uni-scatterer tags case (Fig. 5), we reduced $L$ by $100 \mu \mathrm{m}$ in the design of film mask for group M. Here, too, the natural process variations during the PCB realization are considered as a part of purposely applied variations. A photograph of all tags of both groups along with their dimensions is shown in Fig. 10. The overall size of these fabricated tags is also same as of the simulated ones': $8 \times 15 \mathrm{~mm}^{2}$.

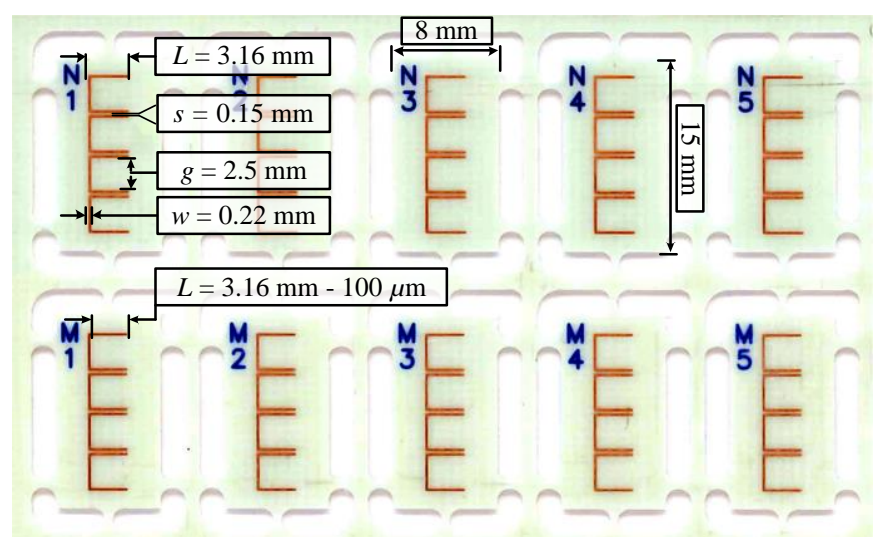

Fig. 10. Top view of the fabricated C-folded quad-scatterers tags along with their dimensions.

The measurement setup for $\mathrm{C}$-folded quad-scatterers tags is shown in Fig. 11, where the measurements are done in a mono cross-polarization configuration using VNA with a source power of $-5 \mathrm{dBm}$ in an anechoic environment. In this configuration, the test tag is placed at an inclination of $45^{\circ}$. The distance between the antenna and the tag is $r=13.5 \mathrm{~cm}$. 
The measured quantity is the transmission coefficient $S_{21}$ as shown in Fig. 12. Similar to the C-folded uni-scatterer tags, five repetitive measurements are taken for each C-folded quad-scatterers tag. For each measurement trial, the tag is removed and repositioned at its position to ensure the validity of repeatability in the presence of an observational error. Furthermore, the background normalization is applied. Then, the time windowing [27] with a time window of $10 \mathrm{~ns}$ is employed to extract the antenna mode, where $2.5 \mathrm{~ns}$ of early part of signal is discarded to neglect the structural mode [Fig. 12(b)].



Fig. 11. Measurement setup for C-folded quad-scatterers tags in a crosspolarization configuration in an anechoic environment.



(a)



(b)
Fig. 12. Time windowing of the transmission coefficient $S_{21}$ of C-folded quad-scatterers tag N1. (a) Frequency domain responses. (b) Time domain responses.

To highlight the potential of C-folded quad-scatterers tags for authentication, a CS map is presented in Fig. 13. The procedure to calculate the CS map for quad-scatterers tags is same as discussed above for the CS map for uni-scatterer tags in Fig. 8. In this case, the calculated reference parameters are $f_{r}^{m}=12.68 \mathrm{GHz}$ and $Q^{m}=98.8$ (i.e., the mean values of extracted $f_{r}$ and $Q$ parameters of group $\mathrm{N}$ ). The centroid to centroid difference of $\mathrm{CS}$ between groups $\mathrm{N}$ and $\mathrm{M}$ is $\Delta \mathrm{CS}_{4}=0.6$. This $\Delta \mathrm{CS}_{4}=0.6$ is very large than $\Delta \mathrm{CS}_{1}=0.13$ (see Fig. 8). Also, for this quad-scatterers case (Fig. 13), the slope of CS is quite sharp along $\Delta f_{r}$ as compared to the uniscatterer case (Fig. 8). This sharpness is due to the increase in $Q$ of tags from the uni-scatterer tag $\left(Q^{m}=27\right)$ to quadscatterers tag $\left(Q^{m}=98.8\right)$. Such a large similarity change is the reason for the choice of these quad-scatterers tags to be employed for authentication.

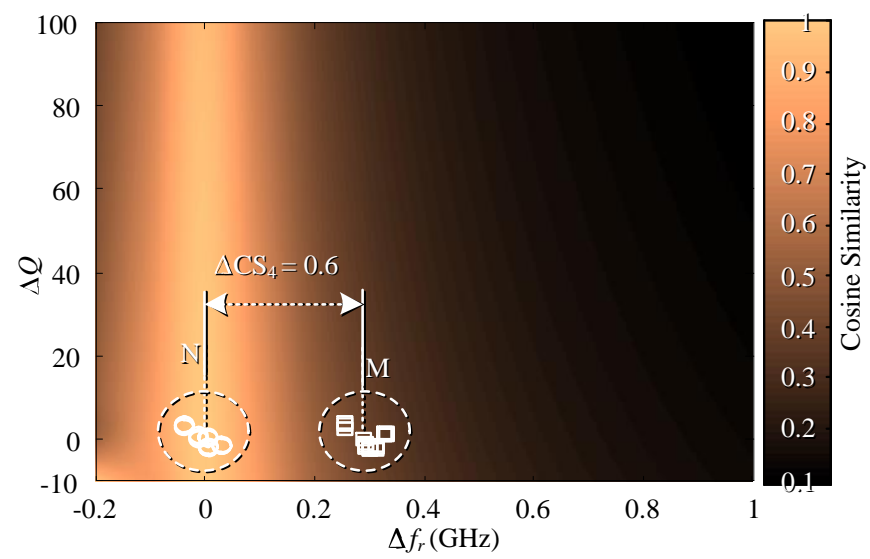

Fig. 13. Cosine similarity map obtained by comparing a reference complex FD signal $x^{r}$ with a varying complex FD signal $x^{v}$ using CS (1) for quadscatterers tags. The superimposed markers $\bigcirc$ and $\square$ correspond to extracted parameters of twenty-five measurements of five tags of $\mathrm{N}$, and $\mathrm{M}$ groups, respectively.

\section{DETECTION OF NATURAL RANDOMNESS AND AUTHENTICATION RESULTS}

To demonstrate the authentication based on solely naturally occurring randomness in the fabrication process, we have realized the chipless tags based on $\mathrm{C}$-folded quad-scatterers. These tags are realized two times intermittently, where each realization is constituted of forty-five tags. These two intermittent realizations share same company, same technology, but different film mask to ensure the natural dimensional randomness. The film masks are formed using the geometrical dimensions of group $\mathrm{N}$ depicted in Fig. 10 (i.e., without any purposely applied variations).

Fig. 14 shows the top view of forty-five tags of first realization exhibiting natural randomness. The frequency of resonance $f_{r}$ of all these tags is around an average value of $12.68 \mathrm{GHz}$ as shown in Fig. 12. For the rest of this paper, the measurement setup and the post-processing (i.e., background normalization and time windowing) of measured signals (i.e., $S_{21}$ ) are also exactly same as depicted in Figs. 11 and 12. Additionally, the time windowing process is one step further improved by applying the frequency windowing on FD windowed signals. This frequency windowing is done by keeping the 200 points around the peak apex while discarding all other points. This procedure is adopted to consider only the aspect-independent parameters $\left(f_{r}\right.$ and $Q$ ) that principally reside in the top part of skirts of peak apex. Hence, FD windowed signal (i.e., also subjected to the frequency windowing) is constituted of 200 points. For TD windowed signal, if we ignore the zero padded points, the signal is constituted of 101 points. The zero padded points can be ignored because they do not contribute to the similarity change. Instead, the zero padding in TD signal is done only to improve the resolution of FD windowed signal while computing the inverse Fourier transform in the time windowing process. For the rest of this paper, we take into account the windowed signals (i.e., also subjected to the frequency windowing) for the similarity analysis. 


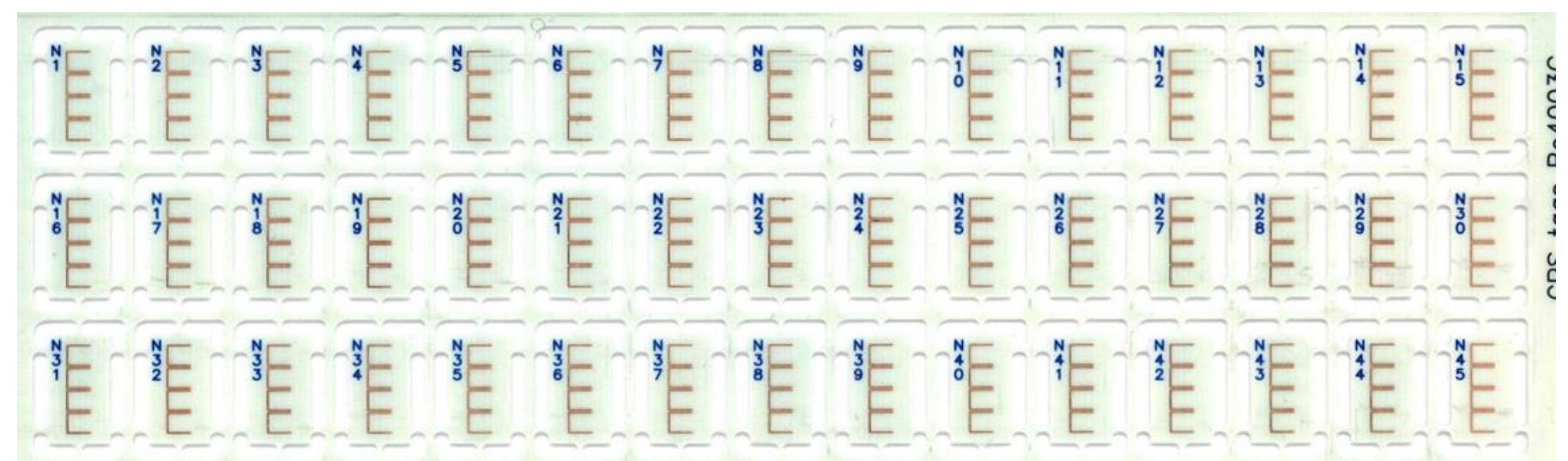

Fig. 14. Top view of first realization of C-folded quad-scatterers tags exhibiting natural randomness.

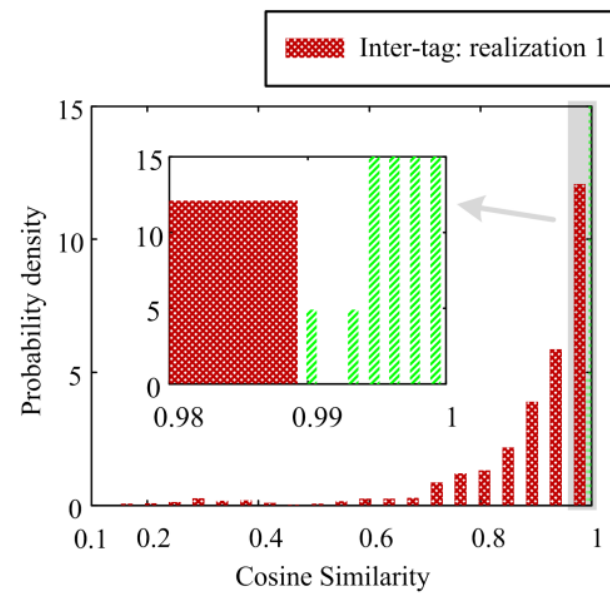

(a)
พIIIIIII. Intra-tag: realization 1



(b)

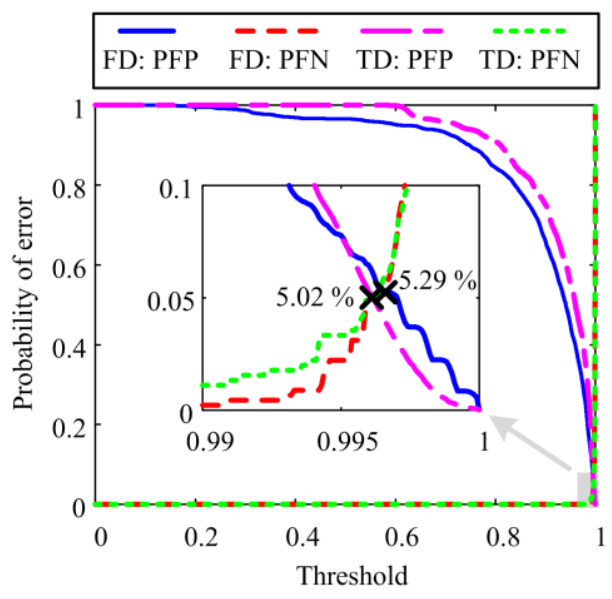

(c)

Fig. 15. Similarity analyses for first realization. (a) Intra- and inter-tag cosine similarity PDFs for FD analysis. (b) Intra- and inter-tag maximum valued correlation coefficient PDFs for TD analysis. (c) Probabilities of false positive and false negative for both FD and TD analyses.

The two realizations are compared within each realization and also between them (see signals' comparison conventions in Fig. 3).

\section{A. Authentication Within Each Realization}

First, the authentication outcomes based on solely naturally occurring randomness within each realization for both intermittent realizations are calculated. For this purpose, all forty-five tags from each realization are measured five times successively. So, the possible number of inter-tag combinations among all different tags within a realization is $C_{2}^{45 \times 5}-45 C_{2}^{5}=24750$ and the possible number of intra-tag combinations to compare all repetitive measurements for all tags from one realization, is $45 C_{2}^{5}=450$. The signal similarity is calculated by using CS (1) and $\mathrm{CC}_{\max }$ (2) for FD and TD signals, respectively, as discussed in Section II.

Fig. 15 shows the intra- and inter-tag $\mathrm{CS}$ and $\mathrm{CC}_{\max }$ similarity PDFs for FD and TD similarity analyses, respectively, along with their PFP and PFN for first realization. The central tendencies of intra-tag similarity PDFs [Fig. 15(a) and (b)] in FD and TD analyses are close to unity with minimum values of 0.989 and 0.986 , respectively. This behavior validates the repeatability of individual tags, i.e. when the same tag is measured multiple times the result is close to a value of 1 . The spreads of inter-tag similarity PDFs [Fig. 15(a) and (b)] are quite wide in comparison to intra-tag similarity PDFs ranging from 0.142 to 0.993 and from 0.587 to 0.996 for FD and TD similarity analyses, respectively. It can be observed that intra- and inter-tag similarity PDFs [Fig. 15(a) and (b)] exhibit overlaps between them, producing PE of 5.29\% and 5.02\% in FD and TD analyses, respectively.

For the authentication results within second realization, Fig. 16 shows intra- and inter-tag $\mathrm{CS}$ and $\mathrm{CC}_{\max }$ similarity PDFs for FD and TD similarity analyses, respectively, along with their error rates (i.e., PFP and PFN). Intra-tag similarity PDFs [Fig. 16(a) and (b)] in FD and TD analyses are centered close to unity with minimum values of 0.99 and 0.988 , respectively. On the other hand, inter-tag similarity PDFs [Fig. 16(a) and (b)] exhibit wide spread ranging from 0.47 to 0.995 and from 0.618 to 0.996 for FD and TD similarity analyses, respectively. In this case too, intra- and inter-tag similarity PDFs [Fig. 16(a) and (b)] exhibit overlaps between them, producing $\mathrm{PE}$ of $5.16 \%$ and $5.93 \%$ in $\mathrm{FD}$ and $\mathrm{TD}$ analyses, respectively.

\section{B. Authentication Across Different Realizations}

All forty-five tags related to each fabrication (see Fig. 14) are realized at the same time on one sample of substrate. From 




(a)
IIIIIIII. Intra-tag: realization 2



(b)

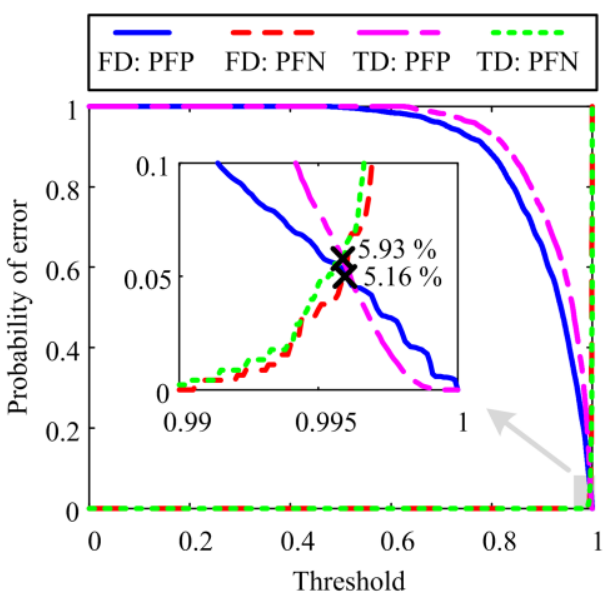

(c)

Fig. 16. Similarity analyses for second realization. (a) Intra- and inter-tag cosine similarity PDFs for FD analysis. (b) Intra- and inter-tag maximum valued correlation coefficient PDFs for TD analysis. (c) Probabilities of false positive and false negative for both FD and TD analyses.

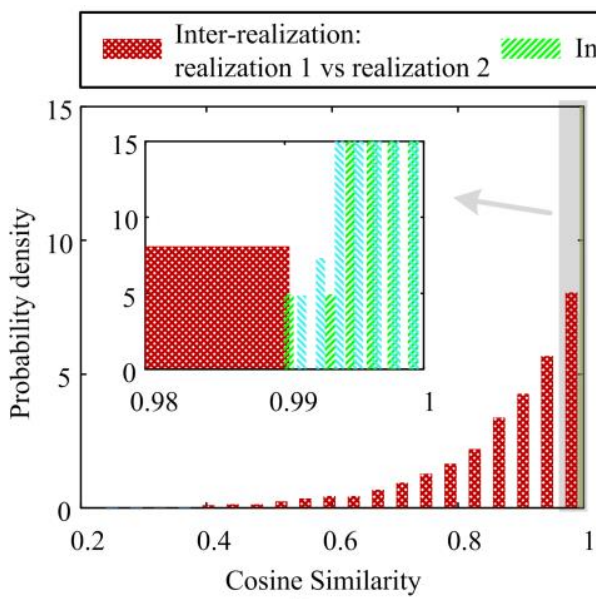

(a)

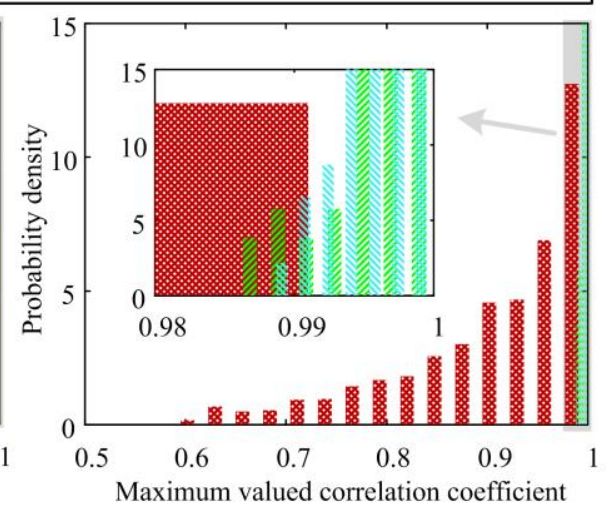

(b)



(c)

Fig. 17. Similarity analyses for first realization vs second realization. (a) Intra-tag and inter- realization cosine similarity PDFs for FD analysis. (b) Intra-tag and inter- realization maximum valued correlation coefficient PDFs for TD analysis. (c) Probabilities of false positive and false negative for both FD and TD analyses.

this fact, the fabrication tolerance would have an almost equal effect on all forty-five structures. So, it is worth to calculate the similarity results across different realizations. For a comparison between two independent realizations, the possible number of inter-realization combinations among all different tags is $(45 \times 5)^{2}=50625$. While the number of intratag combinations for each realization is the same as calculated above, that is, $45 C_{2}^{5}=450$. For the comparison between first realization and second realization, Fig. 17 shows intra-tag and inter-realization CS and $\mathrm{CC}_{\max }$ similarity PDFs for FD and TD similarity analyses, respectively, along with their error rates (i.e., PFP and PFN). In this comparison, the spreads of interrealization similarity PDFs [Fig. 17(a) and (b)] are also quite wide in comparison to intra-tag similarity PDFs ranging from 0.23 to 0.993 and from 0.588 to 0.994 for FD and TD similarity analyses, respectively. The overlaps among intraand inter-realization similarity PDFs [Fig. 17(a) and (b)] produce $\mathrm{PE}$ of $4.06 \%$ and $2.92 \%$ in FD and TD analyses, respectively. Such values of PE are quite low and comparable to the average $\mathrm{PE}$ for fingerprint verification system ranging from $2.07 \%$ to $4.03 \%$ found in [36] and the best average PE of $0.05 \%$ among different fingerprint evaluation campaigns found in [37].

\section{Characterization of the Natural Randomness}

The existence of natural dimensional variations in the realized prototypes of two intermittent realizations is analyzed by microscopic analysis. The measurement setup of a digital microscope is shown in Fig. 18. A Mighty Scope 5M digital microscope by Aven tools is used and the calibration of system is done by the USAF 1951 resolution test chart. The resolution of system is around $3 \mu \mathrm{m}$ per pixel. First, the digital images of chipless tags are captured and then Hough transform is applied to calculate geometrical dimensions in the microscope images.

It is clear from (5) and (7) that $L^{\prime}$ (or alternatively $L$ ) and $g$ are associated with $f_{r}$ and $Q$, respectively. For this reason, eight $L_{m}$ and four $g_{m}$ parameters for all forty-five tags of both intermittent realizations are measured (see inset of Fig. 18). In theory [see (5) and (7)], only one value for each $L^{\prime}$ (or 
alternatively $L$ ) and $g$ parameters is expected. However, in practice, because of quad coupled scatterers, the parameters' values can change from one element to another (see inset of Fig. 18) that can contribute to the randomness of response. Fig. 19(a) shows a comparison of the measured arms' lengths $L_{m}$ for both intermittent realizations along with the value of theoretical arms' length $L$ and Fig. 19(b) shows a comparison of the measured spacings between arms $g_{m}$ for both intermittent realizations along with the theoretical value of the spacing between arms $g$. It can be observed that the mean values of the distributions of both measured parameters $\left(L_{m}\right.$ and $g_{m}$ ) for two realizations are clearly separated. The mean values of the $L_{m}$ for realizations 1 and 2 are $L_{m(\mathrm{R} 1)}=3.13 \mathrm{~mm}$ and $L_{m(\mathrm{R} 2)}=3.16 \mathrm{~mm}$, respectively. The mean values of the $g_{m}$ for realizations 1 and 2 are $g_{m(\mathrm{R} 1)}=2.50 \mathrm{~mm}$ and $g_{m(\mathrm{R} 2)}=$ $2.49 \mathrm{~mm}$, respectively.



Fig. 18. Measurement setup of a digital microscope. Also, the USAF 1951 resolution test chart is shown. In the inset, an example of a tag under test along with its dimensional parameters is shown.

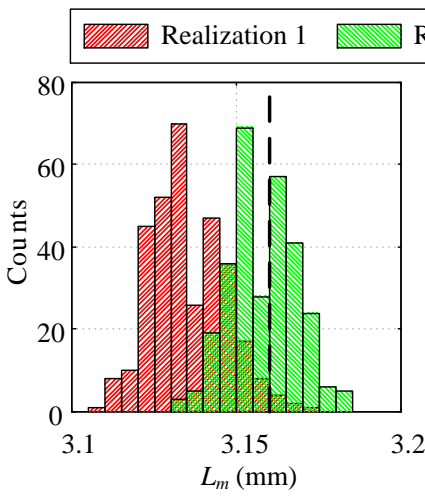

(a)



(b)
Fig. 19. Microscopic dimensional characterization for both intermittent realizations. (a) Measured arms' lengths $L_{m}$ in comparison to the theoretical value of arms' length $L$. (b) Measured spacings between the arms $g_{m}$ in comparison to the theoretical value of spacing between the arms $g$.

Next, we established a relation between the detected natural dimensional variations of $L_{m}$ and $g_{m}$ (see Fig. 19) to the aspect-independent parameters $f_{r}$ and $Q$. The purpose of this relation is to prove that the natural dimensional variations in
PCB realizations are sufficient to provide adequate aspectindependent based similarity variations that do not contain any measurement or systematic noise.

The combinations of two tags from two distributions (see Fig. 19) presenting the extreme variations are detected. These extreme variations are in terms of minimum and maximum average dimensional variations $\left(\Delta L_{m}^{\min }\right.$ and $\left.\Delta L_{m}^{\max }\right)$ and $\left(\Delta g_{m}^{\min }\right.$ and $\Delta g_{m}^{\max }$ ) along $L_{m}$ and $g_{m}$, respectively. A pair of tags, $\left(\mathrm{N} 3_{\mathrm{R} 1}, \mathrm{~N} 3_{\mathrm{R} 2}\right)$, is detected for $\Delta L_{m}^{m i n}$ that consists of $\mathrm{N} 3$ tags from both realizations 1 and 2. For $\Delta g_{m}^{m i n}$, the detected pair of tags, $\left(\mathrm{N} 2_{\mathrm{R} 1}, \mathrm{~N} 15_{\mathrm{R} 2}\right)$, comprises of $\mathrm{N} 2$ tag from realization 1 and N15 tag from realization 2. In contrast, a single pair of tags, $\left(\mathrm{N} 14_{\mathrm{R} 1}, \mathrm{~N} 28_{\mathrm{R} 2}\right)$, is detected for both $\Delta L_{m}^{\max }$ and $\Delta g_{m}^{\max }$, which includes N14 tag from realization 1 and N28 tag from realization 2. For the aforementioned extreme variations, firstly the parameters $f_{r}$ and $Q$ of both tags within each detected pair are extracted from their signals as an average of five repetitive measurements. Secondly, we calculate the minimum and maximum variations $\left(\Delta f_{r}^{\min }\right.$ and $\left.\Delta f_{r}^{\max }\right)$ and $\left(\Delta Q^{\min }\right.$ and $\Delta Q^{\max }$ ) of $f_{r}$ and $Q$, respectively.

Table I outlines the detected pairs of tags from two realizations for $\Delta L_{m}^{\min }, \Delta L_{m}^{\max }, \Delta g_{m}^{\min }$ and $\Delta g_{m}^{\max }$ along with their extracted $\Delta f_{r}^{\min }, \Delta f_{r}^{\max }, \Delta Q^{\min }$ and $\Delta Q^{\max }$. For simplicity, we assume that $\Delta f_{r}$ is merely related to $\Delta L_{m}$ [see (5)] and $\Delta Q$ is merely related to $\Delta g_{m}$ [see (7)]. For this reason, in the cases of $\Delta L_{m}^{\min }$ and $\Delta L_{m}^{\max }$, the values of $\Delta Q^{\min }$ and $\Delta Q^{\max }$ are neglected (highlighted in dark gray) and the values of $\Delta f_{r}^{\min }$ and $\Delta f_{r}^{\max }$ are taken (highlighted in light gray). Similarity, in the cases of $\Delta g_{m}^{\min }$ and $\Delta g_{m}^{\max }$, the values of $\Delta f_{r}^{\min }$ and $\Delta f_{r}^{\max }$ are neglected (highlighted in dark gray) and the values of $\Delta Q^{\text {min }}$ and $\Delta Q^{\max }$ are taken (highlighted in light gray). However, this analysis along with our simplified assumption might only provide the first-order approximation of the results.

TABLE I

Detected Pairs of TAgs From Two Realizations Bases on Extreme DIMENSIONAL VARIATIONS $\Delta L_{m}^{\min } \Delta L_{m}^{\max }, \Delta g_{m}^{\min }$ AND $\Delta g_{m}^{\max }$ ALONG WITH

\begin{tabular}{|c|c|c|c|c|c|c|c|c|}
\hline \multirow{2}{*}{  } & \multicolumn{4}{|c|}{ Minimum variations } & \multicolumn{4}{|c|}{ Maximum variation } \\
\hline & $\begin{array}{c}\text { Pairs of } \\
\text { tags } \dagger\end{array}$ & $\begin{array}{l}\Delta L_{m}^{\min } \\
(\mu \mathrm{m})\end{array}$ & $\begin{array}{c}\Delta f_{r}^{\min } \\
(\mathrm{MHz})\end{array}$ & $\Delta Q^{\min }$ & $\begin{array}{c}\text { Pair of } \\
\text { tags }\end{array}$ & $\begin{array}{c}\Delta L_{m}^{\max } \\
(\mu \mathrm{m})\end{array}$ & $\begin{array}{l}\Delta f_{r}^{\max } \\
(\mathrm{MHz})\end{array}$ & $\Delta Q^{\max }$ \\
\hline $\begin{array}{l}L_{m(\mathrm{R} 1)} \\
\quad \text { vs } \\
L_{m(\mathrm{R} 2)}\end{array}$ & $\begin{array}{l}\mathrm{N} 3_{\mathrm{R} 1} \\
\text { and } \\
\mathrm{N} 3_{\mathrm{R} 2}\end{array}$ & $\approx 0$ & 13.5 & 1.24 & \multirow{3}{*}{$\begin{array}{c}\mathrm{N} 14_{\mathrm{R} 1} \\
\text { and } \\
\mathrm{N} 28_{\mathrm{R} 2}\end{array}$} & 57.5 & 94 & 12.51 \\
\hline$L_{m(\mathrm{R} 2)}$ & & $\begin{array}{l}\Delta g_{m}^{\min } \\
(\mu \mathrm{m}) \\
\end{array}$ & & & & $\begin{array}{c}\Delta g_{m}^{\max } \\
(\mu \mathrm{m})\end{array}$ & & \\
\hline $\begin{array}{l}g_{m(\mathrm{R} 1)} \\
\quad \text { vs } \\
g_{m(\mathrm{R} 2)}\end{array}$ & $\begin{array}{c}\mathrm{N} 2{ }_{\mathrm{R} 1} \\
\text { and } \\
\mathrm{N} 15_{\mathrm{R} 2}\end{array}$ & $\approx 0$ & 0.5 & 2.43 & & 37.4 & 94 & 12.51 \\
\hline
\end{tabular}

$\overline{\dagger \mathrm{N} 3_{\mathrm{R} 1}, \mathrm{~N} 3 \text { tag from realization 1; N3 }{ }_{\mathrm{R} 2}, \mathrm{~N} 3 \text { tag from realization } 2 ; \mathrm{N} 2_{\mathrm{R} 1}, \mathrm{~N} 2}$ tag from realization $1 ; \mathrm{N} 15_{\mathrm{R} 2}, \mathrm{~N} 15$ tag from realization 2.

$\ddagger \mathrm{N} 14_{\mathrm{R} 1}, \mathrm{~N} 14$ tag from realization $1 ; \mathrm{N} 28_{\mathrm{R} 2}, \mathrm{~N} 28$ tag from realization 2. 
Next, the CS map of quad-scatterers tags presented in Fig. 13 is taken as a reference and the chosen values (see the highlighted values in light gray in Table I) are superimposed (in the form of lines) on CS map to create Fig. 20. Fig. 20 describes the range of similarity change that can happen due to the practical natural randomness in comparison to the theoretical model (6) for these quad-scatterers tags. The theoretical model (6) accounts only the aspect-independent parameters (that do not contain any measurement or systematic noise). The superimposed lines form a rectangle enclosing an area of similarity variations on the CS map. The values of CS on the CS map beneath these four vertices of rectangle are $\mathrm{CS}_{v 1}=0.99, \quad \mathrm{CS}_{v 2}=0.99, \quad \mathrm{CS}_{v 3}=0.77$, and $\mathrm{CS}_{v 4}=0.78$, respectively. It is found that the existing natural variations are sufficient to be employed for authentication applications by producing similarity change ranging from $\mathrm{CS}=0.99$ to $\mathrm{CS}=0.77$. It is important to note that these variations are purely based on variations of aspectindependent parameters: $\Delta f_{r}$ and $\Delta Q$. The maximum change in $\mathrm{CS}$ due to the natural randomness $\Delta \mathrm{CS}_{\text {natural }}^{\max }=\mathrm{CS}_{v 1}-\mathrm{CS}_{v 3}=$ 0.22 (Fig. 20) presents same order of magnitude as for the simulated results $\Delta \mathrm{CS}_{4}{ }^{\mathrm{CST}}=0.22$ and $\Delta \mathrm{CC}_{\max 4}{ }^{\mathrm{CST}}=0.23$ (Fig. 9). On the other hand, $\Delta \mathrm{CS}_{\text {natural }}^{\max }$ is less than the change in CS due to purposely applied variation $\Delta \mathrm{CS}_{4}=0.6$ (Fig. 13). The reason for this decrease is that in natural randomness the maximum detected change of $\Delta L_{m}$ is $\Delta L_{m}^{\max }=57.5 \mu \mathrm{m}$ (see Table I), while the purposely applied variation is $100 \mu \mathrm{m}$ in Fig. 13.



Fig. 20. Range of the similarity change happened due to the natural random variations for quad-scatterers $\mathrm{C}$-folded tags.

One can argue that the detected $\Delta f_{r}$ and $\Delta Q$ parameters might be due to the variation of $\varepsilon_{r}$ of the employed substrate (i.e., Rogers RO4003) from one sample to another. For this reason, we conducted simulations for quad-scatterers tags using its nominal dimensional parameters (see Fig. 9) while changing $\varepsilon_{r}$ from 3.38 to $3.38 \pm 0.05$ that is provided by the manufacturer. From simulated results, we found that an average $\Delta f_{r}$ due to changes in $\varepsilon_{r}$ is $\Delta f_{r}^{\Delta \varepsilon_{r}} \approx 62 \mathrm{MHz}$. This value of $\Delta f_{r}^{\Delta \varepsilon_{r}}$ is less than the detected natural $\Delta f_{r}^{\max }=94 \mathrm{MHz}$ (see Table I), which signifies that the detected natural randomness is not merely due to the variation of $\varepsilon_{r}$. As an additional benefit, the variation of $\varepsilon_{r}$ would contribute to the natural randomness to elevate the potential of chipless RFID technology for authentication applications.

\section{Generalization of the Proposed Method}

From Section IV-B, the worst inter-realization PE equals to 4.06\% [see Fig. 17(c)] in CS case. Such a value of PE might be large for the practical implementation of the proposed method. It is important to note that the natural random variations along the geometrical dimensions of two tags belonging to two different realizations are independent. Therefore, this independent nature of the random variations can be exploited to decrease PE by increasing the number of employed scatterers in the design of chipless RFID tags as shown in Fig. 21(a). For this purpose, the arms' length of the further additive quad-scatterers can be decreased by a step value $d L$ to shift their $f_{r}$ to larger values. With a value $d L=$ $100 \mu \mathrm{m}$, it can be observed from Fig. 21(a) that the peak apex related to each scatterer is well separated. This procedure would aid in the reading of all scatterers in the design of chipless RFID tags simultaneously. The exponential decay of PE with an increase in the employed scatterers can be calculated by using the following expression:

$$
\mathrm{PE}=(0.04)^{n}, n=1,2, \ldots N
$$

where $n$ is the number of scatterers to be employed in the design of chipless tags developed for the authentication process. Fig. 21(b) shows an exponential decay of PE with the increase in the number of scatterers using (8). To achieve a PE $=0.05 \%$ found in [37] from the proposed chipless RFID approach three quad-scatterers are required in the design as mentioned in Fig. 21(b).

On the other hand, even if we consider the worst inter-tag $\mathrm{PE}=5.93 \%$ [see Fig. 16(c)], in such case, too, three quadscatterers are required in the design of chipless tags to reach to the $\mathrm{PE}_{[37]}=0.05 \%$. Therefore, for authentication, the employment of chipless tags realized with different samples of substrates using different film masks (inter-realization case) appears to be equivalent to the employment of chipless tags realized with one sample of substrate using one film mask (inter-tag case). However, in practice, the availability of a large population of substrate samples might be difficult. For this reason, the PCB realizations of chipless tags realized on one sample of substrate (or fewer samples) but with different film masks are the optimum choice.

\section{E. Final Remarks on the Constraints}

The experimental results presented in this work are measured in a room-size anechoic environment. However, the box-size anechoic environment depicted in Fig. 2 would provide comparable results because the anechoic environment in either size fulfills the requirement of isolation from outside environment.

One can argue that the cost for the examination of authenticity in the proposed method is high because of the cost of VNA. Such a high cost is in the same range of other 
available highly secure authentication solutions (e.g., biological authentication solutions or x-rays based authentication solutions), where the cost of equipment to examine the authenticity is also high. On the hand, this highcost equipment is not intended for the common consumer to verify the authenticity of to be purchased items. Even this high-cost equipment might not be intended for the goods suppliers, the distributors, the food and drug administration, or the representatives of pharmaceutical companies. Instead, this is for the customs inspectors, the law enforcement organizations and the court of justice, as these highly secure solutions are specifically for the high-end luxury items.



(a)

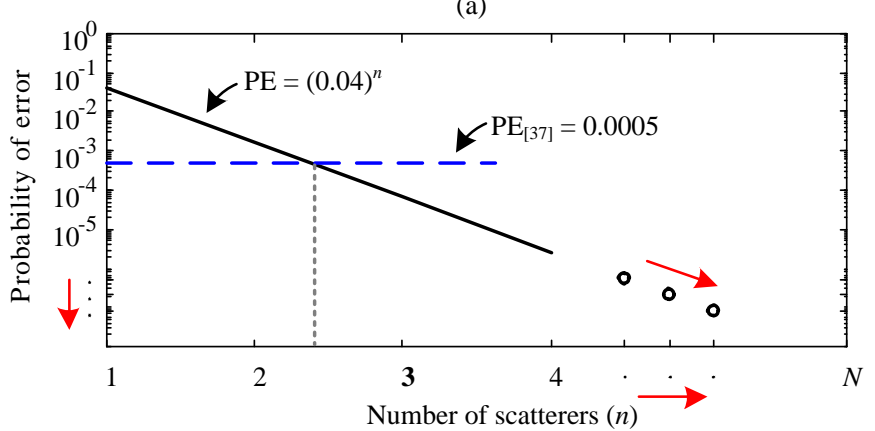

(b)

Fig. 21. Exponential decay of probability of error with the increase in the number of employed scatterers in design of chipless tags. (a) Simulated responses of chipless tags while increasing the number of employed scatterers in design of chipless tags. (b) Exponential decay of probability of error with an increase in the number of employed scatterers in design of chipless tags.

The cost of the system can be reduced by employing the commercially available chipless RFID readers [38], [39]. The impulse radio (IR) ultra-wideband (UWB) reader operates in the frequency band ranging from $3.1 \mathrm{GHz}$ to $10.6 \mathrm{GHz}$ as discussed in [38]. The cost of this reader is comparable with classical RFID reader used in the logistics and the supply chain management. To utilize this IR-UWB chipless reader [38], the action of scaling the design of chipless tags to a lower frequency of operation within UWB poses a slight enlargement in the size of chipless tags. As another alternative, RFIDTECH large reader [39] can also be utilized. The band of operation of this chipless reader is raging from 22 $\mathrm{GHz}$ to $26.5 \mathrm{GHz}$. In this case, the size of chipless tags will be even smaller than presented size in this paper because of the action of scaling the design of chipless tags to a larger frequency of operation, which is advantageous.

\section{CONCLUSION}

In this paper, natural dimensional variations in the design of C-folded tags were analyzed to be utilized for the authentication applications. For this purpose, quad-scatterers tags were chosen because of their sharp slope of dissimilarity. Chipless tags were realized two times intermittently, where each realization was constituted of forty-five tags. Two intermittent realizations share same company, same PCB technology, but different film mask to ensure the natural dimensional randomness. The similarity analyses were conducted inside each realization and also between two intermittent realizations. The achieved probability of error was comparable to various fingerprint evaluation campaigns found in the literature. The existence of natural dimensional variations was also confirmed by the microscopic dimensional analysis for two intermittent realizations. Then, the minimum and maximum changes between the intermittent realizations were also linked to the similarity change to show an intuitive range. It was found that the existing natural variations were enough to produce similarity change to be employed for authentication applications. Finally, the technique was also generalized to decrease the probability of error to a significant level.

\section{REFERENCES}

[1] G. Power, "Anti-counterfeit Technologies for the Protection of Medicines," World Health Organization, Geneva, Switzerland, 2008.

[2] L. Li, "Technology designed to combat fakes in the global supply chain," Business Horizons, vol. 56, no. 2, pp. 167-177, Mar. 2013.

[3] K. V. S. Rao, P. V. Nikitin, and S. F. Lam, "Antenna design for UHF RFID tags: a review and a practical application," IEEE Trans. Antennas Propag., vol. 53, no. 12, pp. 3870-3876, 2005.

[4] S. C. G. Periaswamy, D. R. Thompson, and J. Di, "Fingerprinting RFID Tags," IEEE Trans. Depend. Sec. Comput., vol. 8, no. 6, pp. 938-943, Dec. 2011.

[5] C. Bertoncini, K. Rudd, B. Nousain, and M. Hinders, "Wavelet Fingerprinting of Radio-Frequency Identification (RFID) Tags," IEEE Trans. Ind. Electron., vol. 59, no. 12, pp. 4843-4850, Dec. 2012.

[6] M. Kheir, H. Kreft, I. Hölken, and R. Knöchel, "On the physical robustness of RF on-chip nanostructured security," Journal of Information Security and Applications, vol. 19, no. 4, pp. 301-307, Nov. 2014.

[7] L. Yang, P. Peng, F. Dang, C. Wang, X. Y. Li, and Y. Liu, "Anticounterfeiting via federated RFID tags' fingerprints and geometric relationships," in Proc. IEEE Conf. Comput. Commun. (INFOCOM), Kowloon, Hong Kong, 2015, pp. 1966-1974.

[8] H. P. Romero, K. A. Remley, D. F. Williams, and C. M. Wang, "Electromagnetic Measurements for Counterfeit Detection of Radio Frequency Identification Cards," IEEE Trans. Microw. Theory Techn., vol. 57, no. 5, pp. 1383-1387, May 2009.

[9] B. Danev, T. S. Heydt-Benjamin, and S. Capkun, "Physical-layer Identification of RFID Devices.," in Proc. 8th Conf. USENIX Security Symp., 2009, pp. 199-214.

[10] B. Danev, S. Capkun, R. J. Masti, and T. S. Benjamin, "Towards practical identification of HF RFID devices," ACM transactions on Information and System Security (TISSEC), vol. 15, no. 2, p. 7, 2012. 
[11] H. P. Romero, K. A. Remley, D. F. Williams, C. M. Wang, and T. X. Brown, "Identifying RF Identification Cards From Measurements of Resonance and Carrier Harmonics," IEEE Trans. Microw. Theory Techn., vol. 58, no. 7, pp. 1758-1765, Jul. 2010.

[12] G. Zhang, L. Xia, S. Jia, and Y. Ji, "Identification of Cloned HF RFID Proximity Cards Based on RF Fingerprinting," in Proc. IEEE Trustcom/BigDataSE/ISPA, Tianjin, China, 2016, pp. 292-300.

[13] M. Greene and G. W. Hurley, "Method for forming a radio frequency responsive target and apparatus for verifying the authenticity of same," US6471878 B1, 29-Oct-2002.

[14] J. F. P. Marchand, "Microwave barcode reader using dipole antenna," US6547140B2, 15-Apr-2003.

[15] E. Perret, A. Vena, S. Tedjini, Y. Boutant, and C. Halope, "Item comprising a barcode with an electromagnetic signature," US9697446 (B2), EP2864935 (A2), 2012.

[16] V. Deepu, A. Vena, E. Perret, and S. Tedjini, "New RF identification technology for secure applications," in Proc. IEEE Int. Conf. RFIDTechno. and Applications, Guangzhou, China, 2010, pp. 159-163.

[17] C. Herrojo, J. Mata-Contreras, A. Núñez, F. Paredes, E. Ramon, and F. Martín, "Near-Field Chipless-RFID System With High Data Capacity for Security and Authentication Applications," IEEE Trans. Microw. Theory Techn., vol. 65, no. 12, pp. 5298-5308, Dec. 2017.

[18] F. J. J. Romero, J. V. GARCÍA, L. J. F. FERNÁNDEZ, A. J. Gamo, and B. M. Á. RODRÍGUEZ, "Use of radiofrequency wave absorbing markers for the authentication of security documents," EP2694600B1, 01-Nov-2017.

[19] C. Yang and A. P. Sample, "EM-ID: Tag-less identification of electrical devices via electromagnetic emissions," in Proc. IEEE Int. Conf. RFID, Florida, MA, USA, 2016, pp. 1-8.

[20] K. Yang, D. Forte, and M. M. Tehranipoor, "UCR: An unclonable chipless RFID tag," in Proc. IEEE Int. Symp. Hardw. Oriented Secur. Trust (HOST), Washington, DC, USA, 2016, pp. 7-12.

[21] G. DeJean and D. Kirovski, "Radio frequency certificates of authenticity," US20070005367A1, 04-Jan-2007.

[22] V. Lakafosis et al., "RF Fingerprinting Physical Objects for Anticounterfeiting Applications," IEEE Trans. Microw. Theory Techn., vol. 59, no. 2, pp. 504-514, Feb. 2011.

[23] G. DeJean et al., "RFDNA: A wireless authentication system on flexible substrates," in Proc. IEEE 61st Electron. Compon. and Technol. Conf. (ECTC), Lake Buena Vista, FL, USA, 2011, pp. 1332-1337.

[24] D. F. Christofferson, E. J. Anderson, J. C. Geres, and W. T. Graushar, "Resonator use in the print field," US7506813B2, 24-Mar-2009.

[25] Y. Boutant, J. M. Becker, and T. Fournel, "Method for Extracting Random Signatures from a Material Element and Method for Generating a Decomposition Base to Implement the Extraction Method," CA2953689 (A1), 28-Jun-2007.

[26] Z. Ali et al., "Potential of chipless authentication based on randomness inherent in fabrication process for RF and THz," in Proc. 11th Eur. Conf. Antennas Propag. (EUCAP), Paris, France, 2017, pp. 2559-2563.

[27] Z. Ali et al., "Chipless RFID Tag Discrimination and the Performance of Resemblance Metrics to be used for it," in Proc. IEEE MTT-S Int. Microw. Symp. (IMS), Philadelphia, PA, USA, 2018, pp. 363-366.

[28] Z. Ali et al., "Detection of Minimum Geometrical Variation by FreeSpace-Based Chipless Approach and its Application to Authentication," IEEE Microw. Wireless Compon. Lett., vol. 28, no. 4, pp. 323-325, Apr. 2018.

[29] R. S. Khandpur, Printed Circuit Boards: Design, Fabrication, Assembly and Testing. New York: McGraw-Hill Companies, 2006.

[30] M. Pecht and S. Ganesan, "Open trace defects in FR4 printed circuit boards," Circuit World, vol. 32, no. 1, pp. 3-7, Jan. 2006.

[31] R. Rezaiesarlak and M. Manteghi, "Complex-Natural-Resonance-Based Design of Chipless RFID Tag for High-Density Data," IEEE Trans. Antennas Propag., vol. 62, no. 2, pp. 898-904, Feb. 2014.

[32] E. Perret, Radio Frequency Identification and Sensors: From RFID to Chipless RFID. John Wiley \& Sons, 2014.

[33] R. Garg, I. Bahl, and M. Bozzi, Microstrip Lines and Slotlines, Third Edition: Artech House, 2013.

[34] A. Vena, E. Perret, and S. Tedjini, "Chipless RFID Tag Using Hybrid Coding Technique," IEEE Trans. Microw. Theory Techn., vol. 59, no. 12, pp. 3356-3364, Dec. 2011.

[35] S. Gov and S. Shtrikman, "On isotropic scattering of electromagnetic radiation," in Proc. 18th Convent. Elect. Electron. Eng., Tel Aviv, Israel, 1995, pp. 2.4.4/1-2.4.4/5.
[36] R. Cappelli, D. Maio, D. Maltoni, J. L. Wayman, and A. K. Jain, "Performance evaluation of fingerprint verification systems," IEEE Trans. Pattern Anal. Mach. Intell., vol. 28, no. 1, pp. 3-18, Jan. 2006.

[37] D. Maltoni, D. Maio, A. Jain, and S. Prabhakar, Handbook of Fingerprint Recognition, Second edition. London: Springer-Verlag, 2009.

[38] M. Garbati, E. Perret, and R. Siragusa, Chipless RFID Reader Design for Ultra-Wideband Technology: Design, Realization and Characterization. Elsevier Science, 2018.

[39] "RFIDTECH large reader." [Online]. Available: http://www.rfidtechgroup.com/home/product.html. [Accessed: 18-Jan2019].



Zeshan Ali received the M.S. degree in electrical engineering from King Saud University (KSU), Riyadh, Saudi Arabia, and the Ph.D. degree in optics and radiofrequency from the University Grenoble Alpes, Grenoble, France, in 2015 and 2019, respectively. From 2011 to 2016, he was a researcher with the Department of Electrical Engineering KSU. In 2016, he started his research in the Laboratoire de Conception et d'Intégration des Systèmes (LCIS), Grenoble-INP and focused on the development of chipless RFID systems for product authentication applications. Since 2019, he has been a post-doctoral fellow with the LCIS, Valence, France. His current research interests include chipless RFID, RF signal processing and multi-band filters.

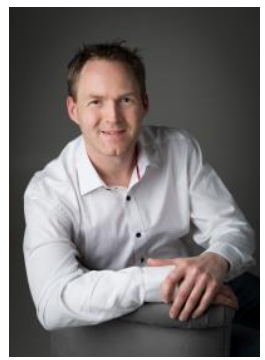

Etienne Perret (S'02-M'06-SM'13) received the Eng. Dipl. degree in electrical engineering from the Ecole Nationale Supérieure d'Electronique, d'Electrotechnique, d'Informatique, d'Hydraulique, et des Télécommunications, Toulouse, France, 2002, and the M.Sc. and Ph.D. degrees in electrical engineering from the Toulouse Institute of Technology, Toulouse, in 2002 and 2005, respectively. From 2005 to 2006, he held a postdoctoral position with the Institute of Fundamental Electronics, Orsay, France. Since 2006, he has been an Associate Professor of electrical engineering with the University of Grenoble Alpes - Grenoble Institute of Technology, Grenoble, France. Since 2014, he has been a Junior Member with the Institut Universitaire de France, Paris, France, an institution that distinguishes professors for their research excellence, as evidenced by their international recognition. Since 2015, he has been an Appointed Member of the French National Council of Universities. He has authored or co-authored over 180 technical conferences, letters and journal papers, and books and book chapters. He holds several patents. His works have generated about 2000 citations. His current research interests include electromagnetic modeling of passive devices for millimeter and submillimeter-wave applications, and wireless communications, especially RFID and chipless RFID, and also include advanced computer-aided design techniques based on the development of an automated codesign synthesis computational approach. Dr. Perret is a Technical Program Committee member of the IEEE International Conference on RFID. He was a recipient of the French Innovative Techniques for the Environment Award in 2013, the SEE/IEEE Leon Brillouin Award for his outstanding achievement in the identification of an object in an unknown environment using a chipless label or tag in 2016 and the IEEE MTT-S 2019 Outstanding Young Engineer Award. He was a Keynote Speaker and the Chairman of several international symposiums. He was named one of the MIT Technology Review's French Innovator's under 35 for his work on chipless RFID in 2013. Etienne Perret was awarded an ERC Consolidator Grant in 2017 for his project ScattererID. 




Nicolas Barbot received the M.S. degree and Ph.D. degree from University of Limoges, France. His Ph.D. work in Xlim laboratory was focused on errorcorrecting codes for the optical wireless channel.

A post-doctoral work was also realized in joint source-channel decoding at the L2S laboratory, at Gif sur Yvette, France.

Since September 2014, he has been an Assistant Professor at Grenoble INP - ESISAR at Valence, France. His research interest at LCIS laboratory includes backscattering communications, RFID, chipless RFID and antenna theory. More recently, he investigates the use of chipless tag as low-cost, batteryless and robust sensors.

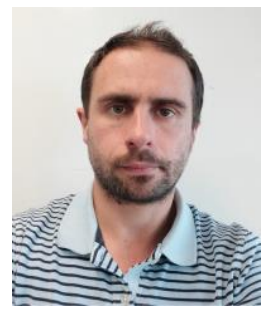

Romain Siragusa was born in Paris, France. He received the engineering and master's degrees from the Institute National Polytechnique de Grenoble, Grenoble Institute of Technology, Valence, France, in 2006, and the Ph.D. degree from the Laboratoire de Conception et d'Intégration des Systèmes, Grenoble Institute of Technology. From 2009 to 2010, he was a Post-Doctoral Fellow with the Commissariat à l'énergie atomique, Saclay, France, where he studied high-impedance surfaces for integrated antennas on silicon. Since 2011, he has been an Associate Professor with LCIS, Esisar, Grenoble Institute of Technology. His current research interests include leaky-wave structures and chipless RFID.

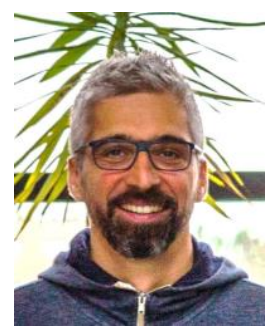

David Hély received the master's degree from the National Institute of Applied Sciences of Lyon, in 2002, and the Ph.D. degree from the University of Montpellier, in 2005, with a focus on the design for testability of secure IC in collaboration with STMicroelectronics and the LIRMM Laboratory. From 2005 to 2009 , he held several positions in research and development with STMicroelectronics and then Sagem Défense et Sécurité, where he was focused on the design and evaluation of secure integrated circuits and embedded systems. Since 2009, he has been an Associate Professor with the Grenoble Institute of Technology, and is a member of the LCIS Laboratory. His research activities span embedded systems cybersecurity including hardware security.

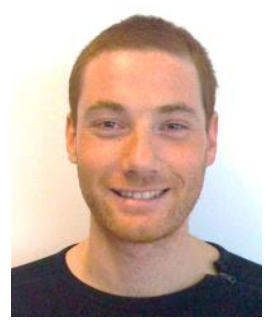

Maxime Bernier was born in France, in 1980. He received a M. Sc. degree in Matter and Radiation from the Burgundy University (France) in 2003, a M. Sc. degree in Microwave and Optical Telecommunications from the University of Limoges (France) in 2004, and his Ph. D. degree in Optic and radiofrequencies in 2008 from the Institut National Polytechnique of Grenoble (France) for a work dedicated to electro-optic detection of E-field. He spent two years as a postdoc fellow within the laboratory of femtosecond spectroscopy at the University of Sherbrooke (Canada). Since September 2011, he is an assistant professor at the IMEPLAHC laboratory, University of Savoy, where his activities are related to $\mathrm{THz}$ signal generation and detection, and accurate materials and devices characterization.

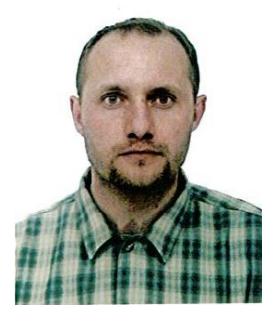

Frédéric Garet was born in France, in 1969. He received the Ph.D. degree in Optics, Optoelectronics and Microwave from Institut National Polytechnique of Grenoble, France, in 1997.

From 1993 to 1997, he was involved in $\mathrm{THz}$ spectroscopy experiment at IMEP-LAHC Laboratory of the "Université de Savoie", France. Since 1998, he's an associate professor at IMEPLAHC Laboratory. His current research interests the Terahertz Time Domain Spectroscopy field, and more precisely precise materials and devices characterization, Thin Films, THz Optics, Plasmonic, and Metamaterials. He is also developing devices for identification and authentication in the $\mathrm{THz}$ domain. 